\title{
Modelling and (Re-)Planning Periodic Home Social Care Services with Loyalty and Non-Loyalty Features
}

\author{
Maria Isabel Gomes ${ }^{\mathrm{a} 1}$ and Tânia Rodrigues Pereira Ramos ${ }^{\mathrm{b}, \mathrm{c}}$ \\ ${ }^{a}$ Centre for Mathematics and Applications (CMA) and Department of Mathematics, \\ Faculty of Sciences and Technology (FCT) \\ Universidade Nova de Lisboa
}

Monte da Caparica, 2829-516 Caparica, Portugal

mirg@fct.unl.pt

${ }^{\mathrm{b}}$ Centre for Management Studies, Instituto Superior Técnico (CEG-IST)

Universidade de Lisboa

Av. Rovisco Pais, 1049-001 Lisbon, Portugal

tania.p.ramos@tecnico.ulisboa.pt

${ }^{\mathrm{C}}$ Business Research Unit (BRU-IUL)

Instituto Universitário de Lisboa (ISCTE-IUL)

Av. Das Forças Armadas, 1649-026 Lisbon, Portugal

tania.ramos@iscte-iul.pt

Please, cite this work as

Gomes, M. I., \& Ramos, T. R. P. (2019). Modelling and (re-) planning periodic home social care services with loyalty and non-loyalty features. European

Journal of Operational Research, 277(1): 284-299

Doi: 10.1016/j.ejor.2019.01.061

${ }^{1}$ Corresponding author: Maria Isabel Gomes, Department of Mathematics, Faculty of Sciences and Technology, 2829-516 Caparica. Tel: (+351)212948388. e-mail: mirg@fct.unl.pt 


\section{Abstract}

The aging population alongside little availability of informal care are two of the several factors leading to an increased need for assisted living support. In this work, we tackle a home social care service problem, motivated by two real case studies where a new loyalty scheme must be considered: within a week, patient-caregiver loyalty should be pursued but, between weeks, the caregivers must rotate among patients (non-loyalty). In addition, a common situation in this kind of service is also addressed: the need of a constant re-planning caused by the leaving of patients and the arrival of new ones. This new plan should be such that minimum disturbance is caused to the visiting hours of current patients, the caregivers' travelling time between visits is minimized, and the workload is balanced among caregivers. A multi-objective optimization approach based on mixed-integer models is developed. Results on the two real case studies show that both institutions can efficiently re-plan their activities without much disturbance on the visits of their patients, and with a patient-caregiver loyalty scheme suiting their needs.

Keywords: OR in health services; Homecare service; (Re-)Routing and (Re-)Scheduling; Non-Loyalty; Mixed-integer linear optimization.

\section{Introduction}

Population aging is a phenomenon faced several countries, mainly western countries, with several social and economic consequences. According to the European Commission (2014), the European Union's population will grow by the 2060-time horizon, but it will be more aged when compared to the present.

As people age their autonomy tends to decrease, leading to the need for support of others to perform their daily living activities. Specialized home social care services providers are a good answer to this need while keeping elders' quality of life and allowing them to continue living in their homes. Home care services providers have increasing concerns regarding the efficiency of their activities to face the current demand (Koeleman et al., 2012). Efficiency is even more important when the service is provided by non-profit organizations, which frequently have tight budgets and need to maximize the productivity of their workforce.

The home social care service is part of the Home Care (HC) service broader concept, which encompasses the providing of medical, paramedical and social services to patients in their homes (Carello and Lanzarone, 2014). Nonetheless, the provision of medical and paramedical services differs from the delivery of social services. While the former is provided by skilled resources (e.g. nurses or medical doctors) to people who are ill or recovering from an illness or injury at home; the latter is 
aimed at people who lack autonomy but may not be ill, usually elderly people. In medical and paramedical services, each patient may require a specific skill from the caregiver; therefore, not all human resources can visit all patients. To provide social services (as bathing, dressing, medication assistance, home cleaning, among others) human resources can be non-skilled workers and so patients can be visited by any caregiver (Gershon et al., 2008). In addition, the devices and consumables needed to assist the patient are different. Regarding medical and paramedical services, caregivers must bring medical instruments and consumables according to the patient's needs. In social services, materials are found in the patient's home and, as such, the caregiver does not have to bring along anything. Consequently, when performing logistics and resource planning of HC systems, those differences must be considered.

Another aspect differentiating $\mathrm{HC}$ planning from other logistics planning problems is the continuity of care, also known as patient-caregiver loyalty. This work is motivated by a request in terms of patientcaregiver loyalty from two non-profit organizations providing social services at patients' homes. The social workers in charge want to plan the caregivers' daily work schedule (which patient to visit and when) for a given time horizon under an unusual (in literature) loyalty scheme: within a week, patientcaregiver loyalty should be pursued, assuring each patient is cared for by the same caregiver during the entire week - loyalty within a week; however, the caregivers must rotate among patients on a weekly basis - non-loyalty between weeks. The goal of this rotation scheme is two-fold: 1) to prevent the appearance of conflicts between caregivers and patients, and 2) to prevent musculoskeletal injuries, very common to this professional group (Olinski \& Norton 2017), since some patients demand a higher level of strength from the caregivers than others. In addition, this type of service faces a very frequent change of patients. Often, there are patients leaving the system (a patient may get too frail and need 24-hour assistance and may go to a nursing home, or may get too sick and need to be hospitalized, or may pass away), and, when possible, care should be provided to new patients from the waiting list. Therefore, almost every week something happens that causes a change of plan, and the social workers wish the new plan does not impact too much on the lives of other patients. Since the demand for these kinds of services surpasses their supply, the social workers need to select the new patients to provide care from a large waiting list. This is not a straightforward task since the needs of the first patient on the waiting list may not fit the available schedule from the patient that have left the system, while the second and third patients' needs might allow a better use of the available time. For instance, they might live nearer to some already visited patients, or they might need services with smaller duration and, therefore, better fitted to the time available. So, to whom should the services be provided? Who does best fit with the current service plan? Notice that elderly people have considerable difficulties in dealing with changes in their everyday life. If they are expecting the 
caregiver to arrive at 10 a.m., many may be disturbed if, next week, the caregiver arrives at 11 a.m. and, in the following week, again at 10 a.m.

Therefore, this work aims at developing a methodological approach based on optimization tools to support the planning of the caregivers' work schedule for several weeks that may be indefinable repeated. Embedded in this approach one has: loyalty between caregivers and patients in each week, a non-loyalty scheme among weeks, and the possibility to re-schedule the existing visiting plan when patients leave the system and new ones must be selected.

This paper unfolds as follows. In section 2, the related literature is reviewed, and the novelty of the proposed work presented. The two case studies that motivated this work are described in detail in section 3. The modelling approach and the MILP models are detailed in section 4 . In section 5 , both case studies are solved with the new models developed and the results are discussed. Lastly, section 6 is dedicated to the concluding remarks and future work directions.

\section{Literature review}

$\mathrm{HC}$ resource planning can be divided into three levels: (i) districting and resource dimensioning, (ii) operator assignment, (iii) scheduling and routing definition (Lanzarone and Matta, 2014). Regarding the operator assignment level, the type of relationship between patient and caregiver is an issue that needs to be addressed. Continuity of care means that, when a caregiver is assigned to a patient, the assignment is to be maintained for as long as possible. Consequently, the patient always receives care from the same caregiver during extended periods. In the opposite side is the non-continuity of care scenario, where the patient can be cared for by different caregivers. In medical and paramedical services, the problem is called nurse-to-patient assignment. Nickel et al. (2012) address the continuity of care as a soft constraint. The authors develop a model to maximize the loyalty between nurse and patient by introducing a penalty in the objective function when a patient is visited by more than one nurse. This is combined with other three objectives (minimize the number of unscheduled tasks, overtime costs, and travelling distance) in a weighted sum objective function. Carello and Lanzarone (2014) consider three distinct types of continuity of care (hard, partial and not required) and two groups of patients (patients under treatment at the beginning of the time horizon and patients starting their treatment during the planning horizon). Patients needing hard continuity of care will not see their reference nurse changed during the time horizon. Partial continuity of care means that the reference nurse can be changed, although it is not desirable. If more nurses are needed, reassignment costs are added to the objective function (like in the approach adopted by Nickel et al., 2012). The 
overall objective is to minimize the total overtime and reassignment costs. The authors use a cardinality-constrained approach to model uncertainty regarding the working time required by each patient. A real case is addressed where 22 nurses must be assigned to about 600 patients living in three different territories. Patients and nurses are divided into two classes: palliative and nonpalliative care. Six districts are considered (one for each class-territory pair) and the assignments are planned independently for each district, in a rolling horizon of 26 weeks. Lanzarone and Matta (2014) also address the nurse-to-patient assignment problem under continuity of care but focus on assigning newly admitted patients. Uncertainty regarding new patients' demand and nurses' workload is also considered. Lin et al. (2016) study the assignment of therapists to patients, where continuity of care, patient's priority and preferred time periods are modelled. A mixed integer programming model is presented and applied to small, medium and large size instances and to a real case study from an HC provider in Hong Kong.

Regarding the scheduling and routing level, daily or weekly plans with visit sequences for each nurse are defined. The daily routing and scheduling problem is addressed, for instance, in the works of Begur et al. (1997), Cheng and Rich (1998), Bertels and Fahle (2006), Eveborn et al. (2006, 2009), Akjiratikarl et al. (2007), Rasmussen et al. (2012), Trautsamwieser et al. (2011), Braekers at al. (2016). In a very recent review of home health care routing and scheduling problems, Fikar and Hirsch (2017) conclude that this single period context is the most studied in the literature. However, the daily planning is a relaxation of the weekly planning, since services are planned only for one day, and cannot handle rest breaks between working days, maximum working time per week and loyalty between nurse and patient (Trautsamwieser and Hirsch, 2014).

The medium-term HC planning problem is addressed in Trautsamwieser and Hirsch (2014), where a Branch-Price-and-Cut algorithm is developed to solve their optimization model. This problem aims at finding nurses' work schedules by assigning patients' visits to each nurse within a week. Different skilled nurses, patient's time-windows, maximum working time per day, breaks during the day and rest times between working days must be respected while minimizing nurses' total working time. The authors generate test instances in accordance with real-life conditions at the Austrian Red Cross, and are able to solve to optimality instances with up to nine nurses, 45 patients and 203 visits during the week.

Another important feature in medium-term HC problems is that patients may require several visits/services per day. Temporal dependencies between visits are studied in the work of Rasmussen et al. (2012): synchronization, overlap, minimum difference and maximum difference. Mankowska et al. (2014) also address interdependent services, considering the scheduling of both single and double services. According to the authors, a double service consists of two services performed by two staff 
members that can be split into simultaneous services or services with a given precedence relation. The authors also considered several visits a day, different time windows preferences, different skills and qualifications levels of staff members and temporal interdependencies of the double services. Three objectives are addressed: minimizing the total daily distance travelled by staff, minimizing the delay of services to reduce patients' waiting times and, finally, to schedule a fair allocation of inevitable waiting times between patients. A MILP formulation and a heuristic procedure are proposed, and test instances results are presented.

A different logistics problem is addressed in the work of Liu et al. (2014). Instead of focusing on the care given to patients, these authors focus on pickup and delivery of materials and goods (such as drugs, medical devices, blood samples) between patients' homes and hospitals, medical laboratories and depots. The problem is modelled as a periodic home health care pickup and delivery problem. The objective is to minimize the length of the longest route in the planning horizon, aiming at workload balance among drivers. Several Tabu Search algorithms are developed and tested on several benchmark instances. A case study based on the French reality is also solved for five different weeks.

In face of this literature review, one may conclude that more attention has been given by the academia to the planning of medical and paramedical services rather than the planning of social services. Moreover, most of works focuses on continuity of care when assigning the caregivers to patients. All published works assume the planning of visits are made from scratch and do not consider the existing visiting schedules. In terms of objectives, most multi-objective problems apply a weight sum approach and do not investigate the possibility of trade-offs among objectives.

This paper further contributes to the home care service literature by addressing two real-world case studies on home social care, and by proposing a modelling and optimization approach that:

- allows the planning of new patients' visits, minimizing disturbance on the current patients' visits and minimizing caregiver travelling time between patients' homes,

- provides the social workers with a weekly visiting plan where daily and weekly loyalty are assured,

- allows for both the optimization of routing and scheduling from scratch or from a previous existing visiting scheme,

- proposes a multi-objective approach where a compromise between travel time minimization and the minimization of disturbance in the current patients' visiting hours is achieved, and also trade-offs the workload balance among teams with the total travel time, 
- defines a weekly rotation scheme for caregivers - non-loyalty -, i.e., defines a multiple week working plan for caregivers where all visit all patients and work with each other according to the rules suggested by the social workers,

- tackles, through the rotation working scheme, the prevention of work injuries by alternating the visited patients and, consequently, the performed tasks,

- develops a working scheme for caregivers with a rolling horizon (i.e., when finishing the last planned week, the rules for teams' composition are observed if the first week is picked as the next one), which allows the caregivers to know in advance exactly what will their working hours be - periodic plan.

\section{Case Studies}

As previously mentioned, this work is motivated by two Portuguese real case studies of home social care service providers. The main services provided by these organizations are (1) home care service, where caregivers go to patients' homes and provide services such as bathing, dressing, diaper changing, medication assistance and home cleaning; (2) meal delivery, where caregivers go to patients' home only to deliver meals; and (3) transportation of patients to day care centre, where caregivers pick the patients up at their homes in the morning and bring them to the day care centre. The caregivers do not have any specific skills that make them neither more nor less suitable to take care of any of the patients. In both case studies, every week the social workers manually plan the work of each caregiver, given the needs of each patient. The two case studies differ in size and capacity to serve patients. The first case concerns a small catholic parish, which takes in 60 patients at the local day care centre, prepares and delivers meals to 66 patients, provides home care service to 17 patients and transport one patient to the day care centre. The second case is a non-profit organization, which handles 50 patients at its day care centre, prepares and delivers meals to 100 patients, provides home care services to 36 patients and transports 4 patients to the day care centre.

In both case studies, social workers expressed their concerns regarding the system "dynamism", where almost every week a few patients leave the system, a long waiting list to manage, and the changing of routines (i.e., visiting hours) of the current patients that must be avoided at least to some less "adaptable" patients to routine changes.

\subsection{Small Catholic Parish - case A}

At the small catholic parish, six caregivers are available to provide the services mentioned above. The caregivers work in teams of two, starting at 8 a.m. until the end of their shift, at 4 p.m. All patients must be visited within this period. The lunch break is between 1 p.m. and 2 p.m. at the day care centre. 
One team must help with the meal delivery, meaning that they must return to the day care centre by 11.30 a.m. This service takes 90 minutes. Given the proximity of patients' houses, the caregivers walk between each house. Patients are served from twice a week to several times per day depending on their needs. The visiting time-windows are defined by the social worker in accordance with the tasks to be performed. For example, home cleaning can be done at any time during the day, while bathing should be done in the morning. The services provided to the patients remain almost unchanged from week to week. Within the week and the day, the team visiting each patient must not change - daily and weekly loyalty.

There are no incompatibility issues between patients and services to be provided and the caregivers, so all caregivers can visit any patients and provide any kind of service. Likewise, there is no incompatibility between caregivers, so any caregiver can pair with any other. Besides that, the social worker specifically requested that all caregivers must pair with all other caregivers at least once during the planning horizon and that all caregivers must visit all patients on a weekly rotation scheme - nonloyalty between weeks. Therefore, each patient is visited by the same team during the week, but to allow continuity of care and the transference of information regarding the patient between caregivers, one of the team members must be the same for at least two to at most three consecutive weeks. The second member of the team must be a different one every week.

\subsection{Non-profit organization - case B}

Nine caregivers work in the non-profit organization and the patients fall into one of two groups: bedridden or semi-dependent. The bedridden patients must be visited by a team of two caregivers, while the semi-dependent patients must be visited by only one caregiver. The social worker has not yet been able to plan the visits in accordance, and, therefore, there are bedridden patients frequently visited by only one caregiver.

Patients' visits can occur from 8 a.m. until 8 p.m. from Monday to Friday. The one-hour lunch break can start between 12 a.m. and 2 p.m. The lunch break period is flexible since not all caregivers have the same working schedule. To help with the meal delivery, three caregivers are needed at the day care centre before 11.30 a.m. and are assigned to this task for a period of 90 minutes. Given the size of intervention area of this non-profit organization, the caregivers travel by car. As in the case of the catholic parish, patients' visits may be twice a week to several times a day. The time-window to visit each patient is also defined by the social worker according to the patient's needs. In this case, each patient should be preferably visited by the same caregiver within a day and within a week. Again, there is no incompatibility between patients/services to be provided and the caregivers. The weekly rotation scheme explained for case A also works for case B. 


\section{Modelling Approach}

The problem in study integrates two different levels of HC resource planning: the operator assignment, and the scheduling and routing definition. In addition, to account for the loyalty feature within a week and the non-loyalty feature between weeks, the model needs to be a multi-period one in a rolling horizon context. Therefore, even for the small-size real case-studies as the ones presented (both in terms of the number of caregivers and patients), the planning period of several weeks will lead to a very large problem and, for sure, too large computational times need to solve it (if not out of memory issues). On the other hand, the minimum number of weeks that allow the rolling horizon is also unknown a priori.

Our strategy was to break down the problem in two separated sub-problems, since the rotation scheme that allocates caregivers to patients and the routing and scheduling decisions can be taken independently, if one assigns teams of caregivers to patients without specifying the caregiver that belongs to such team. Therefore, the routing and scheduling problem concerns the definition of "when" and "by whom" each patient should be visited in each day of the week, with the "by whom" question being answered in terms of "Team 1", or "Team 2". Therefore, instead of defining which caregiver visits which patient and when, we decide which team visits which patient and when and, in parallel, we define which caregiver belongs to which team. When forming the teams, the rotation scheme requested by the social workers is observed. Both problems can be formulated as mixed integer linear models. The "when and by whom" will be based on the periodic vehicle routing problem with time-windows (PVRPTW) where usual constraints of HC problems are added together with a few specific of our problem - team scheduling and routing model. The "team composition" can be formulated as an extension of an allocation model - caregiver assignment model (see Figure 1).

Notice that with this decomposition approach, we can tackle the loyalty and non-loyalty requirements of the social workers without losing solution optimality. In the scheduling and routing model, the loyalty between teams and patients will be imposed allowing the definition of a single week plan. The non-loyalty requirement between weeks, i.e., the rotation scheme of caregivers when forming a team, will be modelled at the caregiver assignment model. Being an independent problem, we will be able to easily determine the minimum number of weeks that allows a scheme with rolling horizon characteristics. 


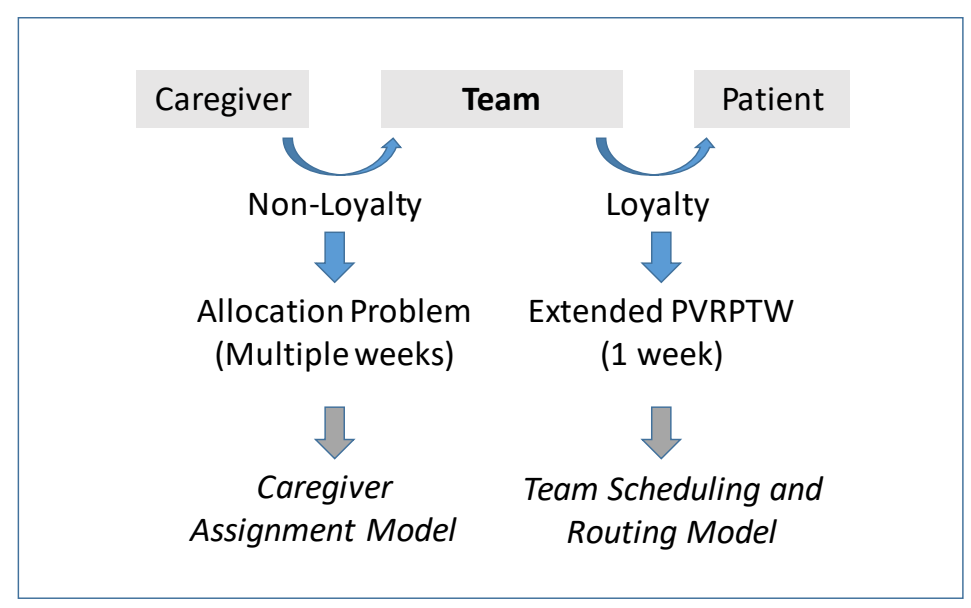

Figure 1: Modelling approach

\subsection{The multi-objective team scheduling and routing model}

Facing the leaving of some patients and the entering of new ones, one set of decisions of this model is to determine the (current and new) patients' week visiting schedule and the visit sequencing for each team of caregivers, without causing much disturbance in the visiting hours of the current patients. A second set of decisions concerns which patient(s) to admit, among those in a waiting list. We have considered that some of the current patients can have their visit starting time changed more than others do. The social workers decide previously which patients have "flexible" and "non-flexible" visiting times. Regarding the objective functions, we have modelled two minimization objectives (total travelling times and total deviation of the visit starting time) and one MinMax objective (balance work among caregivers).

Although the model is developed assuming a pre-existing visiting plan, it can also be used to define scheduling and routing plan from scratch.

\subsubsection{Model formulation}

Having as the PVRPTW a baseline model, a graph $G=(I, E)$ will be defined where patients homes and the day care centre are the nodes (set $I$ ) and the connection between the nodes will define set $E$. For each patient node, a time-window will be defined together with a visiting duration, i.e., the time spent at the patient's home to perform all the required services. The day care centre node is represented with two nodes: 0 and $n+1$. Feasible team visiting routes correspond to paths starting at node 0 and ending in node $n+1$.

By adopting a graph approach, some questions arise concerning the modelling of some of our case distinctive features: (i) how to deal with patients needing more than one visit per day? (ii) how to model patients' transportation from the day care centre? (iii) how to assure that some teams return earlier to the day care centre to help with lunch delivery? and (iv) how to ensure the caregivers' lunch 
break at the day care centre? Our strategy was to replicate all needed nodes. For the patients needing more than one visit, their nodes were replicated as many times as the number of visits and the timewindows were set accordingly. When the service can be provided for a longer period than the maximum working time of caregivers, the daily loyalty has to be relaxed since patients with replicas might have visiting time-windows that are incompatible with one single visiting team. Our strategy was to model the daily loyalty as a soft-constraint, where the assignment of a second team is made possible at the expenses of a penalization in the objective function. For the issues concerning the day care centre, we also replicated its node so that one node would represent meal delivery, another the lunch break and some more the transportation service.

Two additional questions need to be answered: (i) how to model current and new patients? and (ii) how ensure that visit starting time do not change for some patients and might change a bit to others? To address those questions, the modelling strategy focused on the sets definition: (i) patients' set $(P)$ was partitioned into current $\left(P^{\text {cur }}\right)$ and new patients $\left(P^{n e w}\right)$, and (ii) current patients were further partitioned as patient with flexible visit starting times $\left(P^{f}\right)$ and with no flexibility $\left(P^{n f}\right)$.

All the details are given below. We start by defining all sets and indices. Then all parameters are provided, followed by the variables definition. Lastly, the three objective functions and all the constraints are provided.

\section{Indices and Sets}

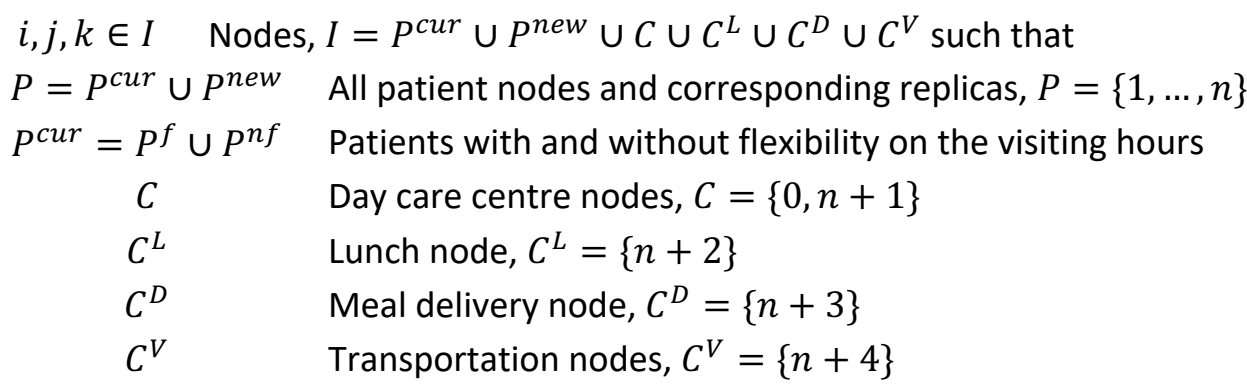

$a \in A \quad$ Caregivers teams

$t \in T \quad$ Planning period (e.g. 5 days of the week)

To model "daily loyalty", we need to link each patient to his/her replicas. So, let $R_{i}^{P} \subseteq P$ be the set of replicas of patient $i$. For the transportation service, we must link the patient node to the transportation node. Consider then set $V \subseteq P$ containing all patients needing transportation.

\section{Parameters}

$d_{i j} \quad$ Shortest travelling time between nodes $i$ and $j$ 


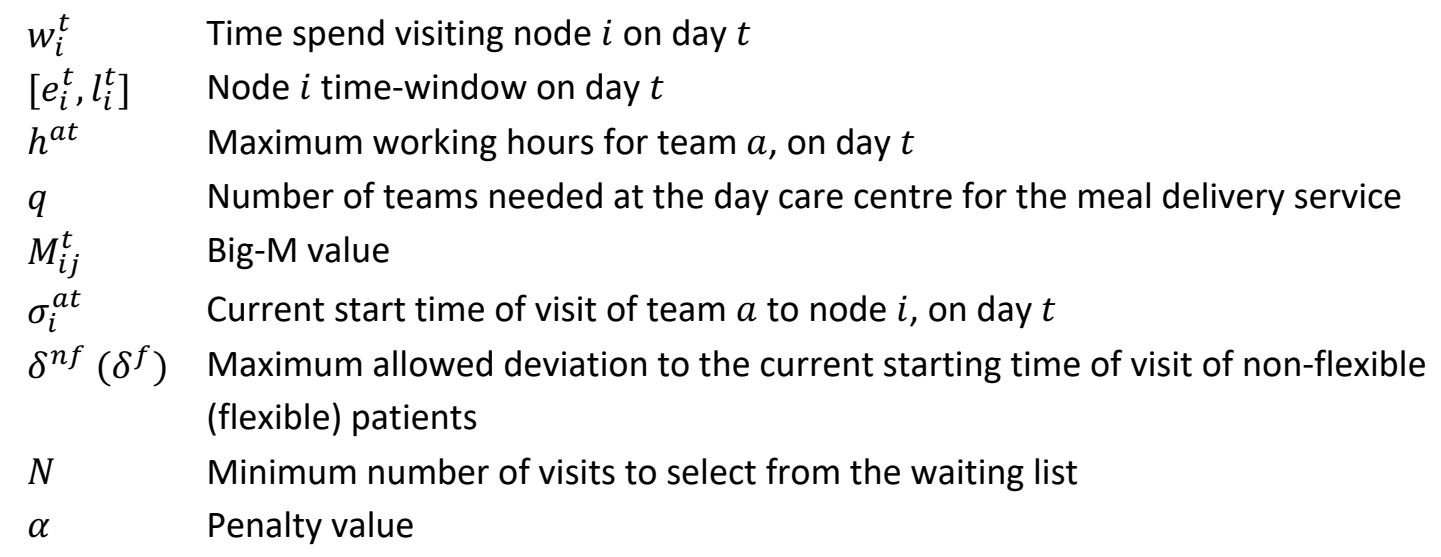

The Big-M value has been defined as $M_{i j}^{t}=\max _{i, j \in P}\left(0, l_{i}^{t}+d_{i j}+w_{i}^{t}-e_{j}^{t}\right)$ as suggested in Cordeau et al. (2007).

Since time windows limit the visiting period, some nodes cannot be visited in sequence. For instance, a patient with a time window at the beginning of the day cannot be visited after a patient whose time window is defined on the afternoon period. In such cases, the direct link between patients $i$ and $j$ has been removed and, consequently, the number of variables decreased. Let $E_{\text {all }}^{T}$ denote the set of all possible direct links between any two nodes on graph $G, E_{\text {all }}^{T}=\left\{(i, j, t): e_{i}^{t}+d_{i j}+w_{i}^{t}-l_{j}^{t}<0 \wedge i \neq\right.$ j\}.

Let $W_{i}$ be the set of the visiting days of patient $i, W_{i}=\left\{t \in T: w_{i}^{t}>0\right\}$, and $\left|W_{i}\right|$ its cardinal.

\section{Variables}

$X_{i j}^{a t}=1$ if team $a$ travels directly from node $i$ to node $j$, on day $t ;=0$, otherwise

$S_{i}^{a t} \quad$ visit starting time of node $i$ by team $a$, on day $t$

$Z_{i}^{a t}=1$ if team $a$ is assigned to node $i$, on day $t ;=0$, otherwise; this is an auxiliary variable that allows an easier modelling of some of the constraints

$Y_{i}^{a t}=1$ if replica $i$ is not visited by the same team as replica $i^{\prime} ;=0$, otherwise

\section{Model formulation}

$$
\begin{aligned}
& \min F_{1}=\sum_{a \in A} \sum_{(i, j, t) \in E_{a l l}^{T}} d_{i j} X_{i j}^{a t}+\sum_{a \in A} \sum_{t \in T} \sum_{j \in R_{i}} \alpha Y_{j}^{a t} \\
& \min \bar{F}_{2}=\sum_{(a, i, t): i \in P^{c u r}}\left|S_{i}^{a t}-\sigma_{i}^{a t}\right| \\
& \min \bar{F}_{3}=\max _{a \in A}\left\{\left(d_{i j}+w_{i}^{t}\right) X_{i j}^{a t}:(i, j, t) \in E_{a l l}^{T}\right\}
\end{aligned}
$$




$$
\begin{aligned}
& \sum_{a \in A} \sum_{(i, j, t) \in E_{\text {all }}^{T}} X_{i j}^{a t}=1, \quad i \in P^{c u r}, t \in T: w_{i}^{t}>0 \\
& \sum_{j \in P \cup C^{L}} X_{0 j}^{a t}=1, \quad a \in A, t \in T \\
& \sum_{j \in P \cup C^{L}} X_{j(n+1)}^{a t}=1, \quad a \in A, t \in T \\
& \sum_{j:(i, j, t) \in E_{\text {all }}^{T}} X_{i j}^{a t}=\sum_{j:(j, i, t) \in E_{\text {all }}^{T}} X_{j i}^{a t}, \quad a \in A, i \in P, t \in T \\
& S_{i}^{a t}+d_{i j}+w_{i}^{t}-S_{j}^{a t} \leq M_{i j}^{t}\left(1-X_{i j}^{a t}\right), \quad a \in A, i, j \in I, t \in T \\
& e_{i}^{t} \leq S_{i}^{a t} \leq l_{i}^{t}, \quad a \in A, i \in P, t \in T \\
& S_{n+1}^{a t}-S_{0}^{a t} \leq h^{a t}, \quad a \in A, t \in T \\
& \sum_{i:(i, j, a, t) \in E_{\text {all }}^{T}} X_{i j}^{a t}=1, \quad a \in A, j \in C^{L}, t \in T \\
& \sum_{a \in A} \sum_{i:(i, n+3, t) \in E_{\text {all }}^{T}} X_{i(n+3)}^{a t}=q, \quad t \in T \\
& X_{i j}^{a t} \leq X_{j k}^{a t}, \quad j \in V, k \in C^{V}, i \in I: i \neq j \wedge i \neq k, a \in A, t \in T \\
& \sum_{j:(i, j, t) \in E_{a l l}^{T}} X_{i j}^{a t}+\sum_{j:\left(i, j, t^{\prime}\right) \in E_{a l l}^{T}} X_{i j}^{a^{\prime} t^{\prime}} \leq 1, \quad i \in P ; a, a^{\prime} \in A: a \neq a^{\prime} ; t, t^{\prime} \in T: t \neq t^{\prime} \\
& \sum_{k:(j, k, t) \in E_{\text {all }}^{T}} X_{j k}^{a t}+Y_{j}^{a t}=\sum_{k:\left(j^{\prime}, k, t\right) \in E_{\text {all }}^{T}} X_{j^{\prime} k}^{a t}+Y_{j^{\prime}}^{a t}, \quad j, j^{\prime} \in R_{i}^{P}, a \in A, t \in T \\
& \sum_{a \in A} \sum_{\substack{(i, j, t) \in E_{\text {all }}^{T} \\
\wedge i \in P^{\text {new }}}} X_{i j}^{a t} \geq N \\
& \sum_{t^{\prime} \in W_{i}} Z_{i}^{a t^{\prime}}=\left|W_{i}\right| \cdot Z_{i}^{a t}, \quad i \in P^{\text {new }}, a \in A, t \in T: w_{i}^{t}>0 \\
& \sum_{a \in A} \sum_{k:(j, k, t) \in E_{\text {all }}^{T}} X_{j k}^{a t}=\sum_{a \in A} \sum_{k:(j, k, t) \in E_{\text {all }}^{T}} X_{(j+1) k}^{a t}, \quad j \in R_{i}^{P} \cap P^{n e w}, t \in T \\
& \sigma_{i}^{t}-\delta^{n f} \leq S_{i}^{a t} \leq \sigma_{i}^{t}+\delta^{n f}, \quad a \in A, i \in P^{n f}, t \in T \\
& \sigma_{i}^{t}-\delta^{f} \leq S_{i}^{a t} \leq \sigma_{i}^{t}+\delta^{f}, \quad a \in A, i \in P^{f}, t \in T \\
& \sum_{j:(j, i, a, t) \in E_{\text {all }}^{T}} X_{j k}^{a t}=Z_{i}^{a t}, i \in P, a \in A, t \in T: w_{i}^{t}>0 \\
& Z_{i}^{a t}=\bar{z}_{i}^{a t}, P^{c u r} \backslash\left\{j: j \in R_{i}^{P}\right\}, a \in A, t \in T \\
& X_{i j}^{a t}, Y_{j}^{a t} \in\{0,1\}, \quad Z_{i}^{a t} \in[0,1], \quad S_{i}^{a t} \geq 0, \quad a \in A, i, j \in I, t \in T
\end{aligned}
$$


The first objective function is the traditional travelling time minimization (function [ 1 ]). We aim at finding the shortest travelling time to visit all patients. Function [ 2 ] minimizes the impact of changing the visiting times for the current patients. This function can be linearized and replaced by function [ 24 ] and constraints [ 25 ] and [ 26].

$$
\begin{aligned}
& \min F_{2}=\sum_{(a, i, t): i \in P^{c u r}} \Delta_{i}^{a t} \\
& S_{i}^{a t}-\sigma_{i}^{a t} \leq \Delta_{i}^{a t}, \quad i \in P^{c u r}, a \in A, t \in T \\
& -S_{i}^{a t}+\sigma_{i}^{a t} \leq \Delta_{i}^{a t}, \quad i \in P^{c u r}, a \in A, t \in T
\end{aligned}
$$

The workload balance objective function is modelled as a MinMax objective, meaning that one aims at minimizing the workload (defined as travel time plus service time) of the team with the heaviest workload (function [ 3 ]). Again, this function is not linear, but can be linearized. To do so, a new variable $U$ is defined, function [ 27 ] replaces function [ 3 ] and constraint [ 28 ] is added to the model. This constraint assures that in each day of the week, teams will work at most $U$ time units.

$\min U$

$\sum_{i j:(i, j, t) \in E_{a l l}^{T}}\left(d_{i j}+w_{i}^{t}\right) X_{i j}^{a t} \leq U, \quad a \in A, t \in T$

If one wishes to impose workload balance over the entire week, the constraint [ 29 ] is the one to add to the model.

$$
\sum_{(i, j, t) \in E_{\text {all }}^{T}}\left(d_{i j}+w_{i}^{t}\right) X_{i j}^{a t} \leq U, \quad a \in A
$$

Equation [ 4 ] assures that all currently visited patients (including all the patients' replicas) are visited exactly once a day, but only on the days the visit has been requested. Equations [ 5 ] and [ 6 ] assure that all teams start their shift at the day care centre (node 0) and finish their shift at the day care centre (node $n+1$ ). Notice that set $C^{L}$ is included in these equations since the lunch break is modelled as fictitious patient visited by all the teams. It is assumed that they can start their shift directly to their lunch break as some teams may only work on the afternoon. Likewise, teams working only mornings can finish their shift at the day care centre immediately after lunch. Equation [ 7 ] models work continuity, i.e. it assures that all teams arrive and leave patients' homes. Constraint [ 8 ] sets the starting time of visit to patients $i$ and $j$ if visited by the same team. When different teams are assigned to patients $i$ and $j$ (i.e. when $X_{i j}^{a t}=0$ ), the corresponding constraint becomes redundant. 
Constraint [ 9 ] assures the time-windows. If team $a$ is available before $e_{i}^{t}$, it is assumed the team will wait so that services does not begin before $e_{i}^{t}$. Being a hard constraint, the team cannot arrive after $l_{i}^{t}$. For the day care centre nodes $(i=0 \vee i=n+1)$ the time windows set the service period. Constraint [ 10 ] defines the maximum working period for team $a$ at day $t$. Constraints [ 4 ] to [ 10 ] are the traditional constraints of a PVRPTW.

Equation [ 11 ] tackles the lunch break, which is modelled as a fictitious patient that all teams must visit. Meal distribution is assured by constraint [ 12 ]. Again, being modelled as special visiting node, it is imposed that at least $q$ teams visit this node in the time window assured by constraint [ 9 ]. This time-window is set by the social worker and can be as small as possible to assure all caregivers arrive on time, i.e., in the worst case the earliest and latest starting times are the same (from 12:00 to 12:00, for example).

Constraint [ 13 ] models the transportation service by imposing that after visiting the patient's node, the day care centre node is the next one to be visited. This constraint does not conflict with the one assuring the team's return at the end of the shift to the day care centre (constraint [6]) since replicas are modelled as different nodes sharing the same location. This replica time-window should be large enough not to limit the final solution, for instance, it could have the length of a working day (e.g., from 8 a.m. until 4 p.m.). With this modelling strategy, one needs to add as many replicas to set $C^{V}$ as the number of patients requesting this service.

Constraint [ 14 ] assures the weekly loyalty by imposing that two teams cannot visit the same patient in two different days of the week. The daily loyalty is imposed by the soft constraint [ 15 ] which is defined for each subset of patients and corresponding replicas.

Constraints [ 16 ] and [ 17 ] impose a minimum of $N$ visits to be selected from the waiting list and assure the admitted patients are visited all days of the week they have asked for. This minimum number is set by the social worker. The need for such constraints comes from the fact that when minimizing any of the given objectives, no patient would ever be selected since it would increase the objective function value. Equation [ 18 ] ensures that if a new patient, having multiple replicas, is selected from the waiting list then all the corresponding replicas are also picked up.

Patients with non-flexible starting times of visits can see their previous starting time $\sigma_{i}^{t}$ change at most $\pm \delta^{n f}$, which can be as small as one or two minutes. This is assured by constraint [ 19 ] which also prevents infeasibilities caused by possible round up (or down) of $\sigma_{i}^{t}$ values. For patients with some time flexibility, a similar approach is used (constraint [ 20 ]). Although they are similar, we opt for making both explicit to reinforce the idea of having two distinct types of changes in the visiting hours. Parameter $\delta^{f}$ is also pre-set by the social worker. If one desires to model flexible visiting time 
for each patient, a parameter $\delta_{i}, i \in P^{c u r}$, would replace $\delta^{n f}$ and $\delta^{f}$, and the two constraints would be merged into one. Notice that since the waiting list and the time flexibility are defined by the social worker, all new patients may eventually enter the system. Equations [ 21 ] and [ 22 ] ensure that current patients are visited by the same team as in the previous planning. Exception is made to the replicas since they might have been visited by different teams (penalization). To finish, constraints [ 23 ] define variables domain. The way the $Z_{i}^{a t}$ variable has been defined within the model (equation [ 21 ]), one can relax its need to be binary. It suffices to impose that $Z_{i}^{a t} \in[0,1]$.

To finish the model definition, we just want to call the reader's attention to the fact that if one wishes to solve a problem ignoring the existence of a previous visiting scheme, constraints [ 16 ] to [22 ] and variable $Z_{i}^{a t}$ should be removed. Also, sub-sets $P^{\text {cur }}, P^{\text {new }}, P^{f}$ and $P^{n f}$ are no longer needed. The resulting team scheduling and routing problem (called HC model, from now on) is an extension of the PVRPTW and therefore is a NP-hard problem. Consequently, we designed a decomposition approach taking advantage of the case studies characteristics and of the previous formulation. Notice that the design of a powerful algorithm to solve this problem optimally is not within the scope of this work.

\subsubsection{Solution Method}

To develop a solution method to solve the $\mathrm{HC}$ model, we looked into it from two perspectives: patient typology and time periods (days of the week). Regarding patient's typology and since different typology means a different team, the separation between bedridden and semi-dependent patients allows for a reduction on instances sizes. This decomposition approach does not impact on optimality since teams working with bedridden patients are different from the teams caring for semi-dependent patients. When considering one day of the week independently from the other, we can further reduce the instance size (Figure 2).

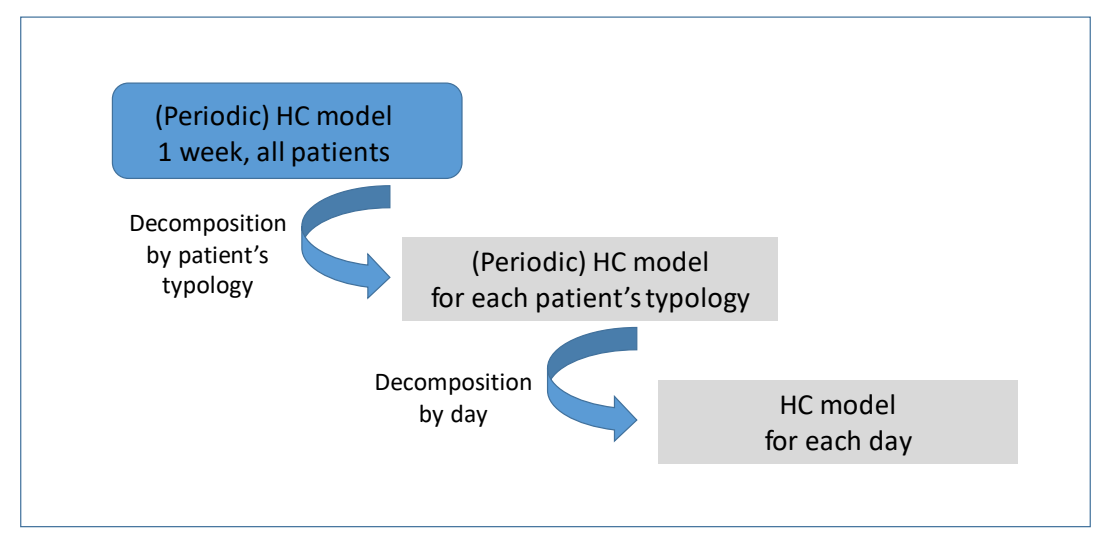

Figure 2: Decomposition approach for the team scheduling and routing problem 
By dealing independently with each time-period, we can no longer assure week loyalty, since constraint [ 14 ] has to be relaxed. A procedure was then developed to assure the final solution met all the requirements. Figure 3 provides its pseudocode.

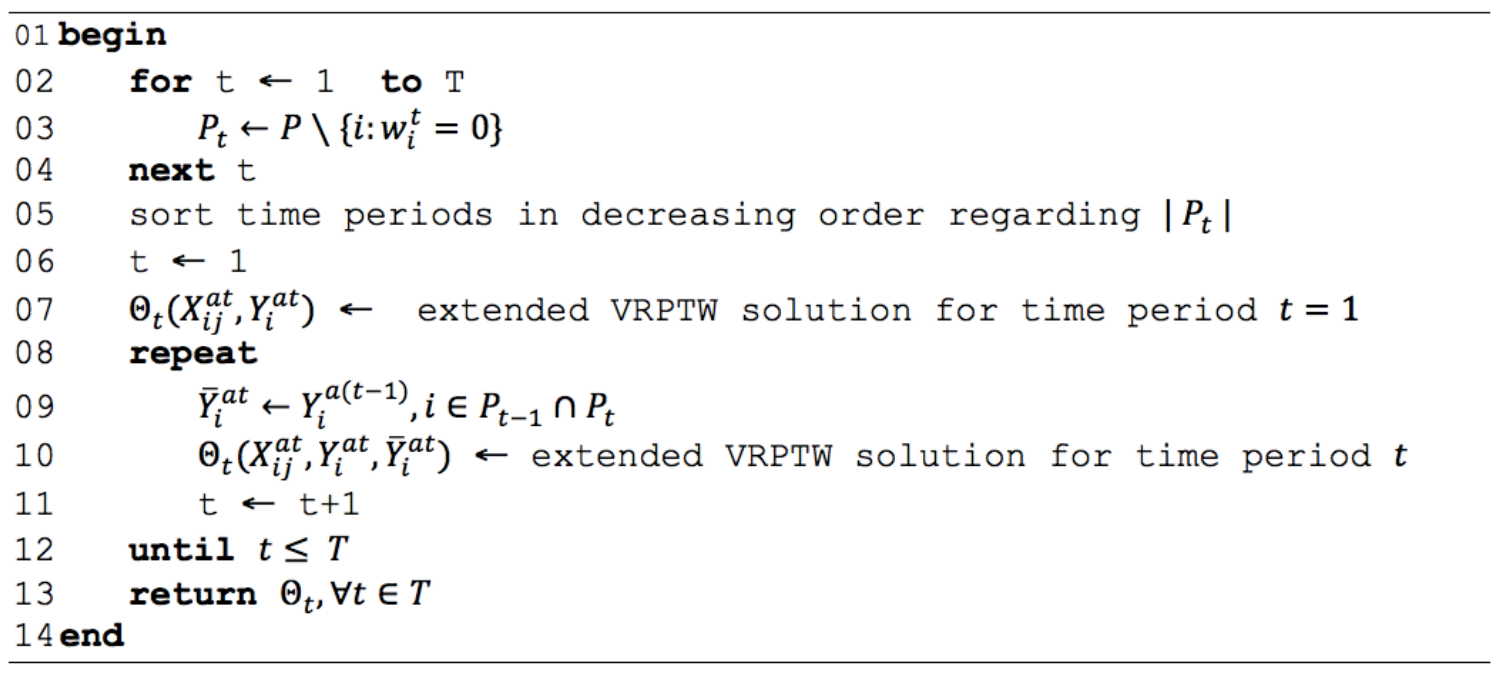

Figure 3: Pseudocode for the heuristic solution procedure of the team scheduling and routing problem

\subsection{Caregiver assignment model}

As mentioned above, the social workers have made some specific requests regarding the way they wanted caregivers to be assigned to patients:

- teams of two, except for the semi-dependent patients of case B that should be visited by only one caregiver,

- all caregivers must pair with all other caregivers at least once during the planning horizon, and

- all caregivers should visit all patients on a week rotation scheme but only one of the team members must be the same for at least two to at most three weeks.

Notice that, while in the previous model a planning horizon is of one week, in this new model, the planning horizon is made of several weeks. To be precise, in this problem, we are not seeking for the optimal assignment since any team structure is good as long as it verifies all the requirements. Rather, we aim at finding the smallest number of time periods allowing the rotation scheme to work on a rolling horizon.

\subsubsection{Model formulation}

\section{Indices and Sets}
$i, j \in I \quad$ Caregivers
$a \in A \quad$ Teams
$t \in T \quad$ Planning period (e.g. weeks)

\section{Parameter}


$k$ Maximum number of consecutive time periods a caregiver may belong to the same team

\section{Variables}

$X_{i j}^{a t}=1$ if caregivers $i$ and $j$ are assigned to team $a$ on time period $t$.

$Y_{i}^{a t}=1$ if caregiver $i$ is assigned to team $a$ on time period $t$.

Given the symmetry inherent to the definition of variable $X_{i j}^{a t}\left(\right.$ since $X_{i j}^{a t}=X_{j i}^{a t}$ ), this variable has only been defined such that $i<j$.

\section{Constraints}

$$
\begin{aligned}
& \sum_{i j: i<j} X_{i j}^{a t}=1, a \in A, t \in T \\
& \sum_{a \in A} Y_{i}^{a t}=1, \quad i \in I, t \in T \\
& X_{i j}^{a t} \leq Y_{i}^{a t}, \quad i, j \in I: i<j ; a \in A, t \in T \\
& X_{i j}^{a t} \leq Y_{j}^{a t}, \quad i, j \in I: i<j ; a \in A, t \in T \\
& \sum_{t^{\prime} \in[t, t+3]} Y_{i}^{a t^{\prime}} \leq k, \quad i \in I, a \in A, t \in T \\
& X_{i j}^{a(t+1)}+X_{i j}^{a t} \leq 1, \quad i, j \in I: i<j ; a \in A, t \in T \\
& X_{i j}^{a t}=1 \Rightarrow Y_{i}^{a(t+1)}=1 \oplus Y_{j}^{a(t+1)}=1, \quad i, j \in I: i<j ; a \in A, t \in T \\
& X_{i j}^{a t}, Y_{i}^{a t} \in\{0,1\}, \quad a \in A, i, j \in I: i<j, t \in T
\end{aligned}
$$

Constraint [ 30 ] assures that each team at each time period is composed of two different caregivers. Equation [ 31 ] assigns each caregiver to exactly one team at each time period. Constraints [ 32 ] and [ 33 ] impose that if caregivers $i$ and $j$ are teamed up at time period $t$ then each of them is assigned to the corresponding team. Constraint [ 34 ] assures that each caregiver is not assign more than $k$ consecutive time periods to team $a$. In consecutive time periods, all teams have to have a different composition (constraint [ 35 ]). The most challenging request to be modelled is the one imposing one team member to be the same at least two consecutive time periods, while the second member must be a different one. Formally, this request can be expressed by relation [ 36 ] where $\oplus$ stands for exclusive disjunction. Expression [ 36 ] can be linearized by constraints [ 38 ] and [ 39 ].

$$
\begin{aligned}
& Y_{i}^{a(t+1)}+Y_{j}^{a(t+1)} \geq X_{i j}^{a t}, \quad i, j \in I: i<j ; a \in A, t \in T \\
& Y_{i}^{a(t+1)}+Y_{j}^{a(t+1)} \leq 2-X_{i j}^{a t}, \quad i, j \in I: i<j ; a \in A, t \in T
\end{aligned}
$$

Lastly, constraint [ 37 ] defines variables domain. 


\subsubsection{Extension to deal with the particularities of case study B}

The model formulation proposed above assumes that all teams have two caregivers. However, in case $B$, the number of caregivers on each team depends on the patient's needs, as only one caregiver visits the semi-dependent patients. To extend the model to account for this feature, we must assign a fictitious caregiver to the single-member teams so that all teams remain comprised of two elements. By adopting this approach, a few changes have to be made to the previous formulation.

Set $A=\left\{1, \ldots, n^{t}, n^{t}+1, \ldots, n\right\}$ will be sorted so that the first $n^{t}$ teams are teams of two caregivers and the remaining $n-n^{t}$ are teams with only one caregiver. Set $A$ can then be partitioned as $A=$ $A_{1} \cup A_{2}$. Let $n^{f}=n-n^{t}$ be the number of fictitious caregivers. Set $I=\left\{1, \ldots, n^{f}, n^{f}+1, \ldots, m\right\}$ should also be sorted so that the $n^{f}$ fictitious caregivers are the last elements of the set. As for set $A$, set $I$ can be partitioned as $I=I_{1} \cup I_{2}$, where $\left|I_{2}\right|=n^{f}$.

Constraints [ 30 ] to [ 33 ] remain unchanged. Constraints [ 34 ], [ 35 ], [ 38 ] and [ 39 ] should now be defined only over set $A_{1}$. Lastly, two new constraints are added. The first one, constraint [ 40 ], assures that each caregiver works as part of teams of two elements no more than twice the number of time periods they work in teams comprised of one caregiver. With this constraint, we are modelling a more balanced assignment among caregivers in terms of the patient's typology, which has been our option to model towards the prevention of musculoskeletal injuries among caregivers. Constraint [ 41 ] assures that the teams with fictitious caregivers do have the same composition after three consecutive weeks, i.e., caregivers cannot be assigned to the same single-member team more than two consecutive weeks.

$$
\begin{aligned}
& \sum_{t \in T} \sum_{a \in A_{1}} Y_{i}^{a t} \leq 2 \sum_{t \in T} \sum_{a \in A_{2}} Y_{i}^{a t}, i \in I_{1} \\
& X_{i j}^{a(t+2)}+X_{i j}^{a(t+1)}+X_{i j}^{a t} \leq 2, \quad i, j \in I: i \neq j ; a \in A_{2}, t \in T
\end{aligned}
$$

\section{Results}

In this section, we analyse and discuss the results obtained with the developed models for both case studies. We first address the multi-objective team scheduling and routing model (section 5.1) where we solve three scenarios (detailed below) and perform a complementary analysis regarding the workload balance and the weekly loyalty vs. non-loyalty impact. The caregiver assignment results are presented in section 5.2. The models have been implemented using GAMS/CPLEX (built 24.4.3) on Intel ${ }^{\circledR}$ Core $^{\mathrm{TM}}$ i5-5200U with $2.2 \mathrm{GHz}$. 


\subsection{The multi-objective team scheduling and routing model}

Data from both case studies was collected and analysed. For the current patients $\left(P^{c u r}\right)$, the social workers provided us with the "service sheets" of each caregiver/team for each day of a given week. Those "service sheets" contain information about the visited patients, starting and ending times of the visits and type of services provided. From those sheets, we were also able to gather information about the patients' service duration $\left(w_{i}^{t}\right)$. Each patient's time windows $\left[e_{i}^{t}, l_{i}^{t}\right]$ were provided by the social workers. From the patients' addresses, we built the distance matrix using the API Google Maps. Given a travel speed (on foot, for case A, and by car, for case B), the distance matrix was transformed into a traveling time matrix $\left(d_{i j}\right)$. For the patients in the waiting list $\left(P^{n e w}\right)$, the data collection process was similar, although an exception was made for the service duration, which was estimated by the social workers. Figure 4 shows the location of $P^{\text {cur }}$ (dots) and $P^{\text {new }}$ (crosses) for both cases.

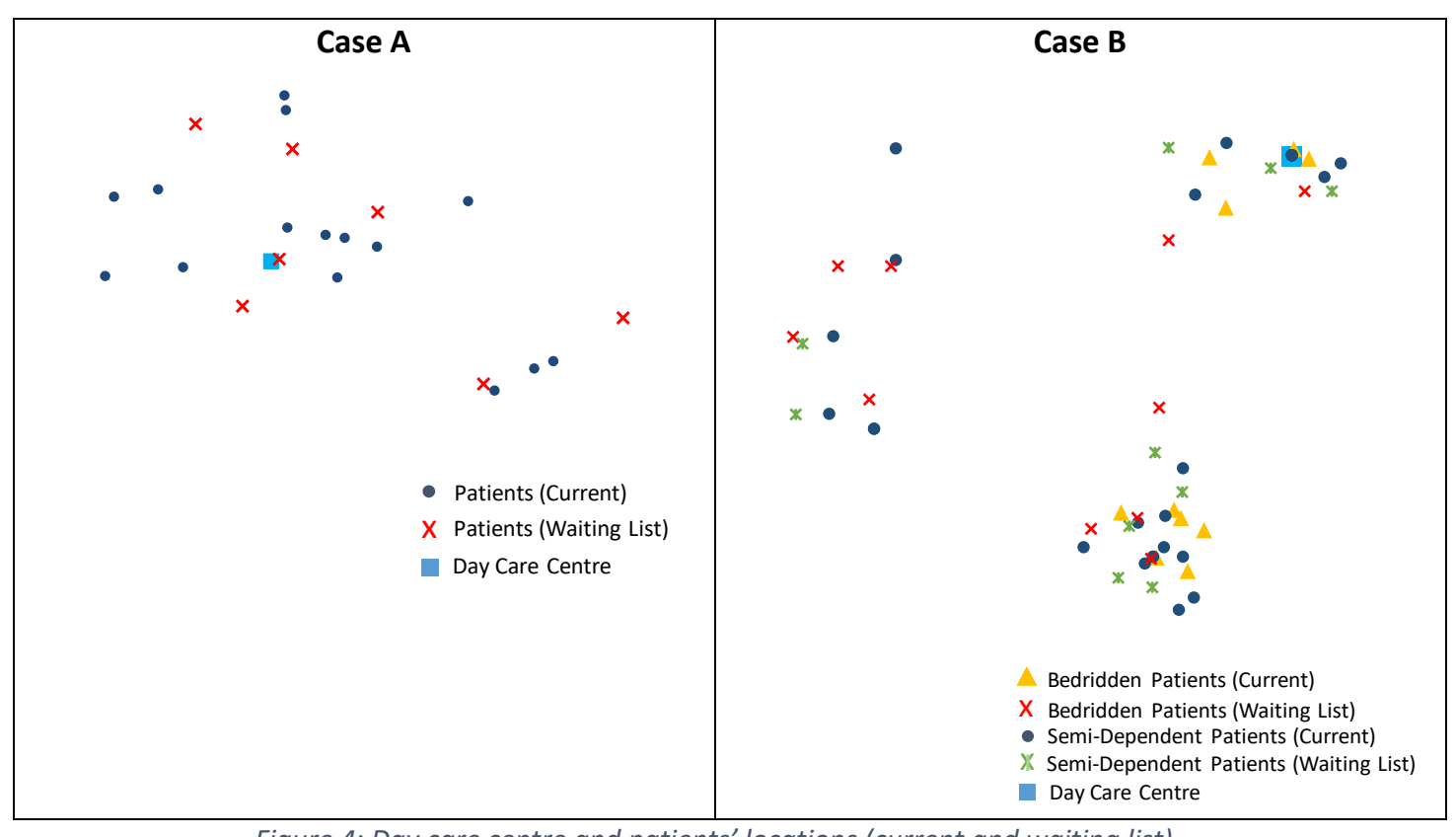

Figure 4: Day care centre and patients' locations (current and waiting list)

From the "service sheets", we were also able to analyse the current patient-caregiver/team assignment and to estimate the current travelling time. For case $A$, three service areas had been defined (one for each team), corresponding to a total of 924 minutes walked per week. Those service areas are depicted in Figure 5, and one can easily observe the possibility of a total walking time reduction. 


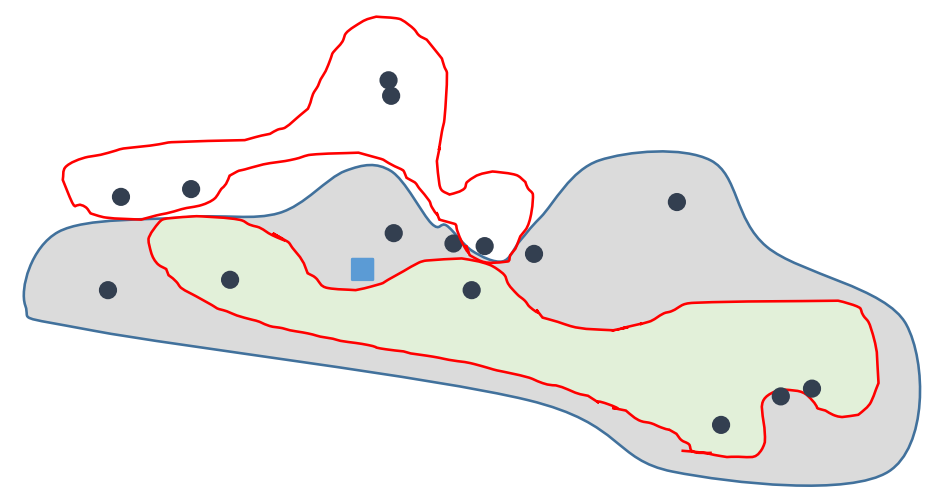

Figure 5: Current service areas for case A

For case B, no service areas can be depicted from the "service sheets" since patients' assignments to teams changed daily (e.g., on Monday two teams were assigned to bedridden patients while on Tuesday there were three teams assigned). The estimated current travel time is 1365 minutes per week. A deeper analysis of these "service sheets" showed that some constraints had not been verified: i) not all the bedridden patients had been visited by teams of two caregivers, and ii) the same patient were visited by different teams along the week, i.e., weekly loyalty was not ensured.

Given the issues that the actual plan presents for both case studies, instead of using these plans to feed our multi-objective team scheduling and routing model, we decide to use an optimized plan, where all constraints are ensured (Scenario 1). Moreover, both social workers stated that the service is at "full capacity", meaning that the caregivers could not visit any more patients. However, we also decided to check if with an optimized plan, more patients could be visited (Scenario 2). Finally, the plan from scenario 2 will be the one feeding the model to select new patients to enter (from the waiting list), when patients eventually leave the system (Scenario 3).

\subsubsection{Scenario 1: Optimized Scheduling and Routing Plan}

To optimize the current week plan, only the $\min F_{1}$ objective function was considered and the solution method presented in section 4.1.2 was applied as the full model failed to provide a good solution for the smaller-size case (case A) - out of memory after 4 hours, best feasible solution with $31 \%$ gap. Therefore, the number of patients (including replicas) to be visited each day of the week was assessed to determine the order by which the solution method would be applied (see Table 1).

Table 1: Number of patients to be visited at each day of the week for cases A and B

\begin{tabular}{lccccc}
\hline \multicolumn{1}{c}{$\boldsymbol{P}^{\text {cur }}$} & Mon & Tue & Wed & Thu & Fri \\
\hline Case A & 22 & 20 & 20 & 22 & 20 \\
\hline Case B & & & & & \\
- Bedridden & 22 & 22 & 22 & 22 & 22 \\
- Semi-Dependent & 20 & 15 & 14 & 14 & 22 \\
\hline
\end{tabular}


For case A, Monday and Thursday have the largest number of patients and the patients are the same, meaning that Monday is equal to Thursday; the same situation occurs for Tuesday, Wednesday and Friday, meaning that the solution method for case A has only 2 iterations:

- $1^{\text {st }}$ Iteration: solve HC model for Monday/Thursday;

- $\quad 2^{\text {nd }}$ Iteration: solve HC model for Tuesday/Wednesday/ Friday, with an imposed patient-team assignment according to the solution provided by the $1^{\text {st }}$ iteration.

The results for case $A$ are presented in Table 2. The total walking time improved the current time by $23 \%$ (714 minutes vs. 924 minutes). With this optimized plan, the teams of caregivers spend $10 \%$ of their time walking between patients' houses, $63 \%$ in providing care to the patients and the remaining $27 \%$ of the working period is idle time due to the waiting to comply with the patients' time-windows.

\begin{tabular}{l|c|c|c|}
\multicolumn{4}{|c|}{ Table 2: Results for Case A (Scenario 1) } \\
& \multicolumn{3}{|c|}{ Case A } \\
& $\mathbf{1}^{\text {st }}$ Iteration & $\mathbf{2}^{\text {nd }}$ Iteration & Week \\
Mon and Thu & Tue, Wed and Fri & Total \\
OFV (min) & 144.7 & 141.6 & 714.2 \\
Computational Time (s) & 740.0 & 1.3 & 741.3 \\
GAP (\%) & 0.0 & 0.0 & - \\
Walking Time (WT) & 144.7 & 141.6 & 714.2 \\
Service Time (ST) & 940.0 & 885.0 & $4,535.0$ \\
Workload (WT+ST) & $1,084.7$ & $1,026.6$ & $5,249.2$ \\
Idle Time & 355.3 & 413.4 & $1,950.8$
\end{tabular}

For the bedridden patients of case B, the same number of patients is observed from Monday to Friday. In this case, we notice that although the patients are the same, some differences exist in terms of duration and time-windows amongst the days of the week. We choose to start the solution procedure with Monday and then, each day of the week was solved imposing the patient-team assignment previously obtained. In total, five iterations were performed. For the semi-dependent patients, also five iterations were made with the following order: $1^{\text {st }}$ Friday, $2^{\text {nd }}$ Monday, $3^{\text {rd }}$ Tuesday, $4^{\text {th }}$ Thursday and $5^{\text {th }}$ Wednesday.

The results for case B are shown in Tables 3 and 4. A total travelling time of 1,280 minutes per week is required to visit both bedridden and semi-dependent patients, resulting in a $6 \%$ improvement when compared to the current solution. Although one might feel that only a small improvement has been achieved, one should notice that the current solution does not meet all the constraints. In our optimized plan, all bedridden patients are visited by teams of two caregivers, with three teams being dedicated to these patients, and the remaining three caregivers are dedicated to the semi-dependent patients. In addition, the weekly loyalty is assured, meaning that along the week patients are always visited by the same team. For the bedridden patients, in some cases the daily loyalty could not be met 
given the duration of a work-shift ( 8 hours) and the several visits needed along the day to a patient (e.g., at 8 a.m. and at 7 p.m.). Given the results in Table 3, there are two patients that could not be visited by the same team within a day (see Penalties row). Regarding the way teams spend their time, comparable results to Case A are observed for the bedridden patients. However, for the semidependent patients, the idle time reaches almost $40 \%$ of the work-shift time (Table 4).

Table 3: Results for Case B - Bedridden Patients (Scenario 1)

\begin{tabular}{|c|c|c|c|c|c|c|}
\hline & \multicolumn{6}{|c|}{ Case B: Bedridden } \\
\hline & $1^{\text {st }}$ Iteration & $2^{\text {nd }}$ Iteration & $3^{\text {rd }}$ Iteration & $4^{\text {th }}$ Iteration & $5^{\text {th }}$ Iteration & Week \\
\hline & Mon & Tue & Wed & Thu & Fri & Total \\
\hline OFV (min) & $4,138.4$ & $4,138.0$ & $4,137.2$ & $4,138.0$ & $4,138.4$ & $20,689.9$ \\
\hline Penalties & 2 & 2 & 2 & 2 & 2 & 10 \\
\hline Computational Time (s) & 23.0 & 0.5 & 0.5 & 0.4 & 0.5 & 24.9 \\
\hline GAP (\%) & 0.0 & 0.0 & 0.0 & 0.0 & 0.0 & 0.0 \\
\hline Travelling Time (TT) & 138.4 & 138.0 & 137.2 & 138.0 & 138.4 & 689.9 \\
\hline Service Time (ST) & 849.0 & 789.0 & 780.0 & 794.0 & 813.0 & $4,025.0$ \\
\hline Workload (TT+ST) & 987.4 & 927.0 & 917.2 & 932.0 & 951.4 & $4,714.9$ \\
\hline Idle Time & 452.6 & 513.0 & 522.8 & 508.0 & 488.6 & $2,485.1$ \\
\hline
\end{tabular}

Table 4: Results for Case B - Semi-Dependent Patients (Scenario 1)

\begin{tabular}{|c|c|c|c|c|c|c|}
\hline & \multicolumn{6}{|c|}{ Case B: Semi-Dependent } \\
\hline & $2^{\text {nd }}$ Iteration & $3^{\text {rd }}$ Iteration & $5^{\text {th }}$ Iteration & $4^{\text {th }}$ Iteration & $1^{\text {st }}$ Iteration & Week \\
\hline & Mon & Tue & Wed & Thu & Fri & Total \\
\hline OFV (min) & 121.1 & 114.8 & 104.9 & 112.9 & 136.3 & 590.0 \\
\hline Computational Time (s) & 0.4 & 0.4 & 0.1 & 0.4 & 2.0 & 3.3 \\
\hline GAP (\%) & 0.0 & 0.0 & 0.0 & 0.0 & 0.0 & 0.0 \\
\hline Travelling Time (TT) & 121.1 & 114.8 & 104.9 & 112.9 & 136.3 & 590.0 \\
\hline Service Time (ST) & 758.0 & 755.0 & 722.0 & 715.0 & 796.0 & $3,746.0$ \\
\hline Workload (TT+ST) & 879.1 & 869.8 & 826.9 & 827.9 & 932.3 & $4,336.0$ \\
\hline Idle Time & 560.9 & 570.2 & 613.1 & 612.1 & 507.7 & $2,864.1$ \\
\hline
\end{tabular}

\subsubsection{Scenario 2: Full Capacity Scheduling and Routing Plan}

Given the optimized plan obtained for both cases studies (Scenario 1) and the fraction of idle time (idle time represents about $30 \%$ of the total working time), the team scheduling and routing model will be used to check if it is possible to include additional patients from the waiting list. For that, the optimized plan from Scenario 1 will be set as the current plan, and the model will select patients from the waiting list to be admitted to the week plan according to parameter $N$. In this scenario, all current patients are considered non-flexible patients regarding the starting time of the visit, with $\delta^{n f}=5$ minutes. The results for both cases are shown at Figure 6 . 


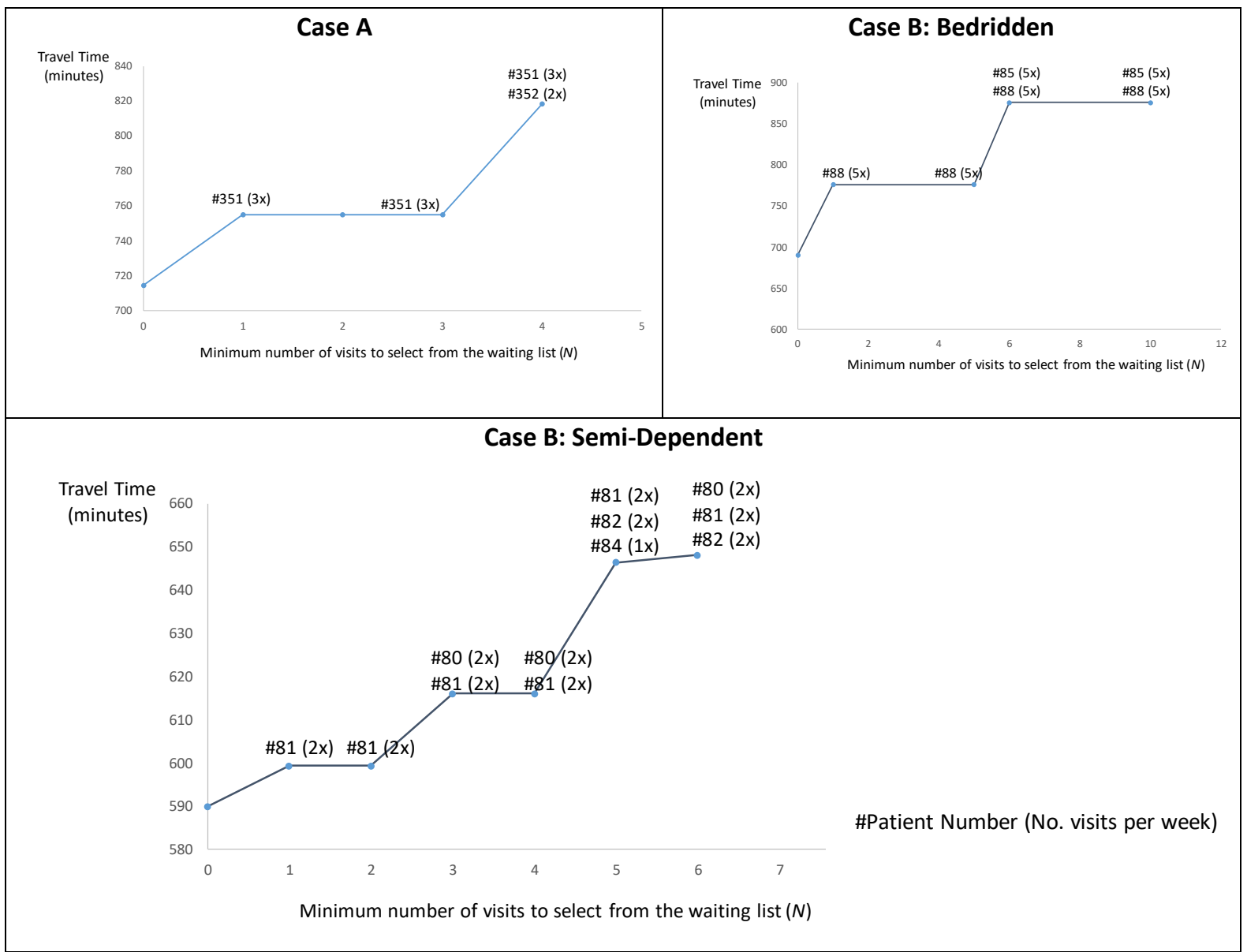

Figure 6: Increase of travel time due the admission of new patients as function of $N$

For case $\mathrm{A}$, it is possible to add a maximum of 5 new visits, corresponding to two additional patients (\#351 and \#352). The model chooses the patients with the smallest number of visits per week. All remaining patients from the waiting list had five or more visits per week. In the full capacity plan, the total walking time increases by $15 \%$, when compared with the optimized plan (714 minutes vs. 818 minutes).

For the bedridden patients (case B), it is possible to add 10 new visits (corresponding to two more patients - \#85 and \#88 -, the one needing only one visit per day). The travel time increases $27 \%$ (from 690 to 876 minutes). When considering $N=11$, no solution exists. It is worth mentioning that both patients were assigned to team 2, which was the team with the largest idle time.

As an illustrative example, Figure 7 shows the detailed routing plan for team 2, on Monday, in Scenario 1 (Optimized Plan) and Scenario 2 (Full Capacity Plan). It can be observed that, for instance, patient 57 is visited at minute 72 in the optimized plan and at minute 67 in the full capacity plan (with a difference of 5 minutes). Moreover, patient 85 was inserted where there was a waiting time period of 50 minutes 
in the optimized plan. The lunch break also changed from starting at around $12.30\left(276^{\prime}\right)$ to start at 2 p.m. $\left(360^{\prime}\right)$.

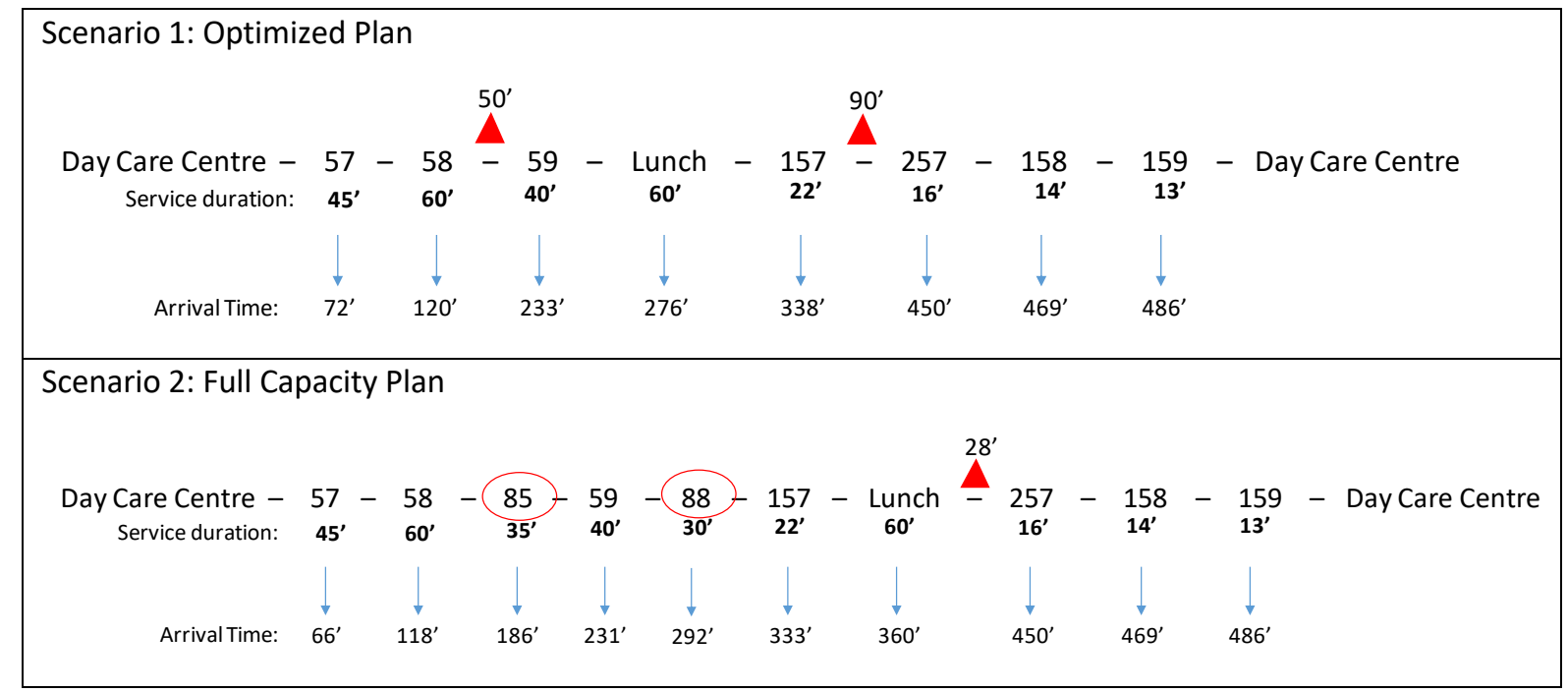

Figure 7: Routes details of team 2, bedridden patients of case B (scenarios 1 and 2)

For the semi-dependent patients, a maximum of 6 visits can be added to the optimized plan without disturbing the visiting hours of the current patients. These six visits correspond to three new patients - patients 80,81 and 82 - and all of them require two visits per week. The total travel time increases 10\% (from 590 to 648 minutes).

\subsubsection{Scenario 3: Re-Scheduling and Re-Routing Plan}

Given the full capacity plan from scenario 2 , where some patients from the waiting list are now being served, scenario 3 will re-do the plan when patients leave the system and new ones can be added. The current patients are divided into flexible patients $\left(P^{f}\right)$, with $\delta^{f}=60$ minutes, and non-flexible patients $\left(P^{n f}\right)$, with $\delta^{n f}=5$ minutes. We simulate the leaving of one patient in a given week, for both cases, using two objective functions: Min Deviation $\left(F_{2}\right)$ and Min Travel Time $\left(F_{1}\right)$. The multi-objective problem is solved through a lexicographic approach. First, a solution is obtained with $\min F_{2}$, and then a solution is obtained for $\min F_{1}$ with an additional constraint imposing a limit on the first objective function $\left(F_{2} \geq \varepsilon \cdot F_{2}^{*}, \varepsilon>0\right)$. With such an approach, we aim at finding a solution that minimizes the travel time compromising at most the deviation in $\varepsilon \%$.

We present below some results for Case B to illustrate the adequacy of the model to tackle the problem. Results for case A are not shown for sake of paper length. The computational times in scenario 3 are negligible, since all simulations were run in just few seconds, proving the optimality of the solutions. 
For the semi-dependent patients, in $85 \%$ of simulations, when one patient leaves the system, it is possible to admit one new patient. In the remaining $15 \%$, two new patients can be admitted. Notice that this depends on the location of both the leaving and the new patients, as well as on the timewindows and on the length of the service of the new patient when compared to the "space freed" by the leaving one.

Figure 8 presents the results in terms of travel time (blue dots) and time deviation (orange dots) from the initial plan, when just one patient leaves the system, and at least one patient must be added $(N=$ 1). The red line indicates the travel time obtained in scenario 2 (full capacity). For example, when patient \#9 leaves the system, patient \#78 (not shown) from the waiting list can be admitted. This leads to a small decrease in travel time $(-0.5 \%)$, and a time deviation of 71 minutes from the initial starting time of visit (two flexible patients changed the visiting hours by 33 minutes each, and one non-flexible patient changed the visiting hour by 5 minutes). From this simulation, we see that almost all cases present a travel time higher than the red line. Although there were (most times) a one to one "replacement", one should not forget that the previous visiting scheme has been optimized considering the leaving patient and not the new one.

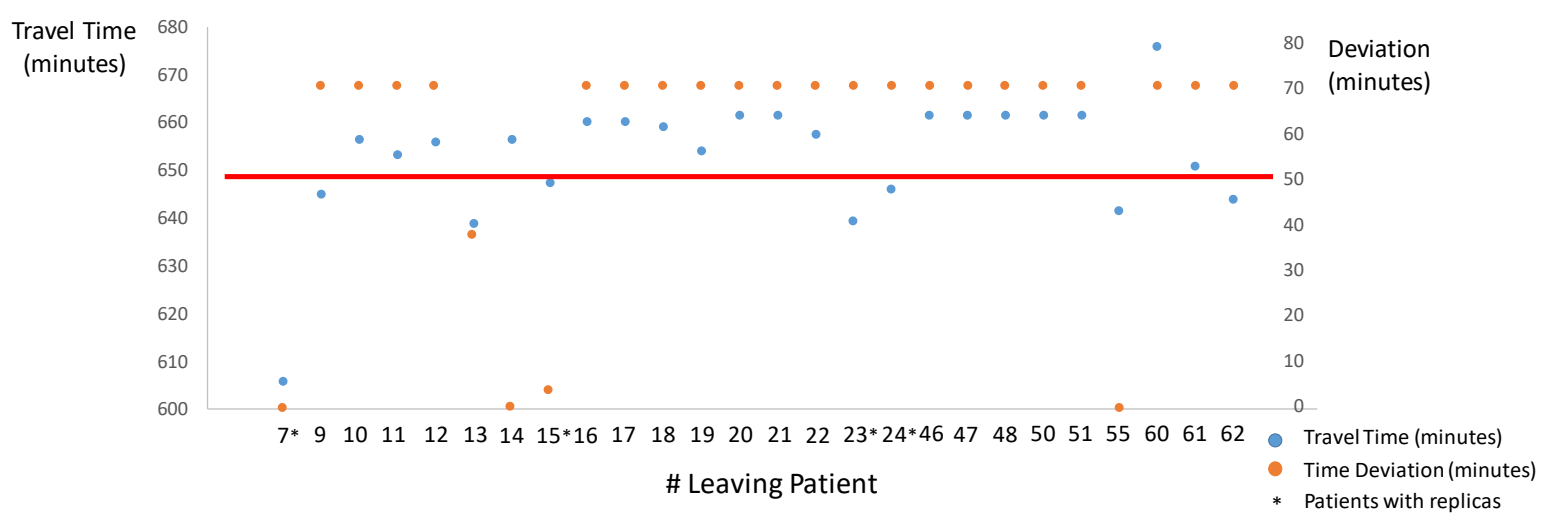

Figure 9: Simulation results for the semi-dependent patients, by leaving patient

The at least one-to-one "replacement" observed for the semi-dependent patients is not observed when considering the bedridden patients. Table 6 shows the simulation results for the leaving of each one of the 10 bedridden patients (that are visited from once a day, e.g., patient \#1, to four times a day, e.g., patient \#2). One can see that in $50 \%$ of those simulations it was not possible to admit any of the patients on the waiting list (given the values defined for $\delta^{f}$ and $\delta^{n f}$ ). Moreover, in certain cases, the number of penalties increases considerably, meaning that more violations are being made to the daily loyalty. The impact on the travel time is not significant, varying from $-6 \%$ (when patient \#59 leaves and patient \#87 is admitted) to $+3 \%$ (when patient \#2 leaves and patient \#86 is admitted). 
Table 6: Simulation results for the semi-dependent patients, by leaving patient

\begin{tabular}{|c|c|c|c|c|} 
Patient Out (\#) & Travel Time & Penalties & Time Deviation & Patient In (\#) \\
Baseline & 876.1 & 10 & - & - \\
1 & 902.2 & 10 & 4.3 & $87,87^{\prime}, 87^{\prime \prime}$ \\
$2,2^{\prime}, 2^{\prime \prime}, 2^{\prime \prime \prime}$ & 894.7 & 10 & 96.7 & $86,86^{\prime}, 86^{\prime \prime}, 86^{\prime \prime \prime}$ \\
$3,3^{\prime}, 3^{\prime \prime}$ & 868.3 & 11 & 7.1 & $87,87^{\prime}, 87^{\prime \prime}$ \\
4 & $N A$ & $N A$ & $N A$ & $N A$ \\
$5,5^{\prime}, 5^{\prime \prime}$ & $N A$ & $N A$ & $N A$ & $N A$ \\
$6,6^{\prime}$ & $N A$ & $N A$ & $N A$ & $N A$ \\
56 & $N A$ & $N A$ & $N A$ & $N A$ \\
$57,57^{\prime}, 57^{\prime \prime}$ & $N A$ & $N A$ & $N A$ & $N A$ \\
$58,58^{\prime}$ & 896.7 & 15 & 9.6 & $87,87^{\prime}, 87^{\prime \prime}$ \\
$59,59^{\prime}$ & 821.8 & 15 & 0 & $87,87^{\prime}, 87^{\prime \prime}$
\end{tabular}

\subsubsection{Complementary Analysis}

After a scheduling and routing plan has been set up for each team for a given week, meeting all the constraints that were imposed by the social workers and fulfilling the objectives of minimizing the travel time (for the optimized and full capacity plans) and the time deviation to the initial plan (when patients leave the system), we will now address two concerns. The first one pertains to a human resources management perspective, where the workload of each team will be assessed and a new plan will be defined so that the workload is balanced among teams. The second one regards the service provided to the patients and intends to assess the impact of "breaking" the loyalty constraints within a week (daily and weekly).

\section{- Workload}

The weekly workload for each team was assessed for the optimized plan (Figure 9). It can be observed that minimum walking/travelling time solutions are quite unbalanced in both case studies.

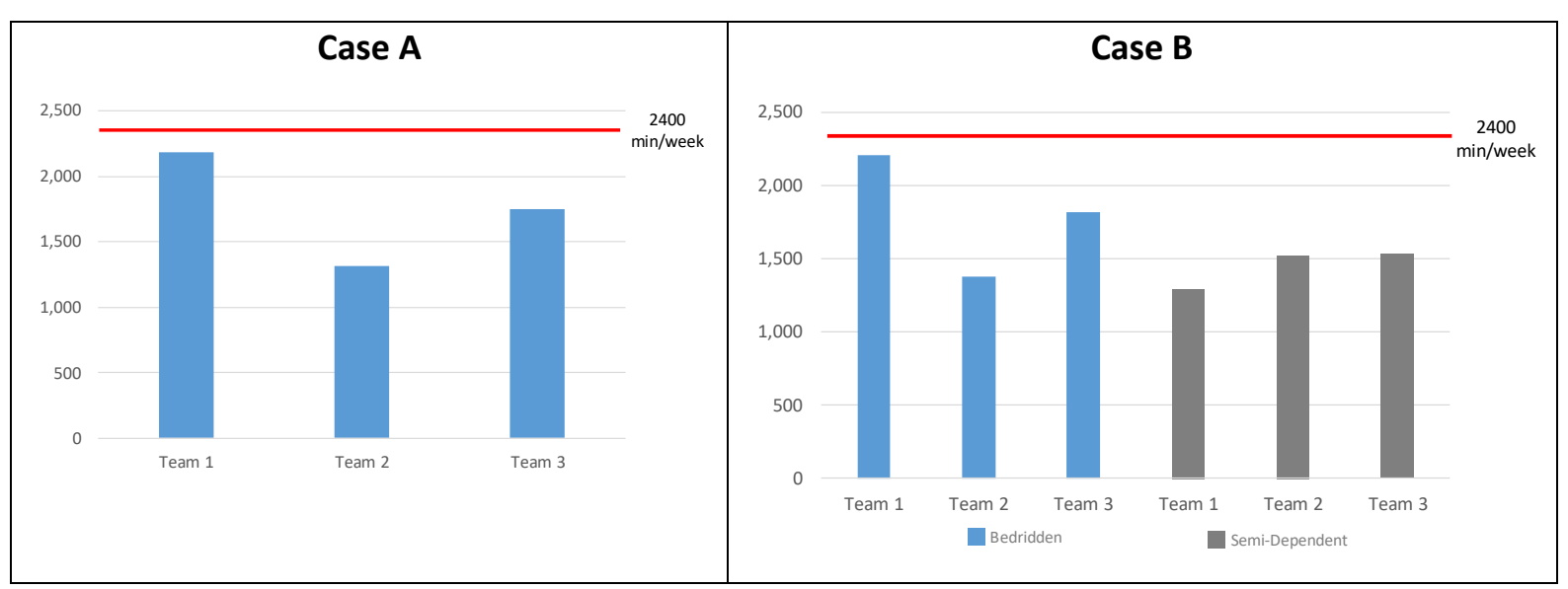

Figure 9: Workload of each team in scenario 1

Therefore, the team scheduling and routing model is now run with the objective function $\min F_{3}$, by using the lexicographic approach described above considering as the second objective function $\min F_{1}$ 
and $\varepsilon=1.1$. For both cases, balanced solutions are found, where all teams work almost the same number of hours per week (Figure 10). However, more balanced workload solutions can be obtained at the expenses of the travelling time. For case $A$, the walking time increases by $10 \%$, from 714 $\mathrm{min} /$ week to $785 \mathrm{~min} /$ week. For case B, an increase of $8 \%$ is observed for the semi-dependent patients (from $590 \mathrm{~min} /$ week to $636 \mathrm{~min} /$ week) and only $2 \%$ for the bedridden patients (from $690 \mathrm{~min} /$ week to $704 \mathrm{~min} /$ week).

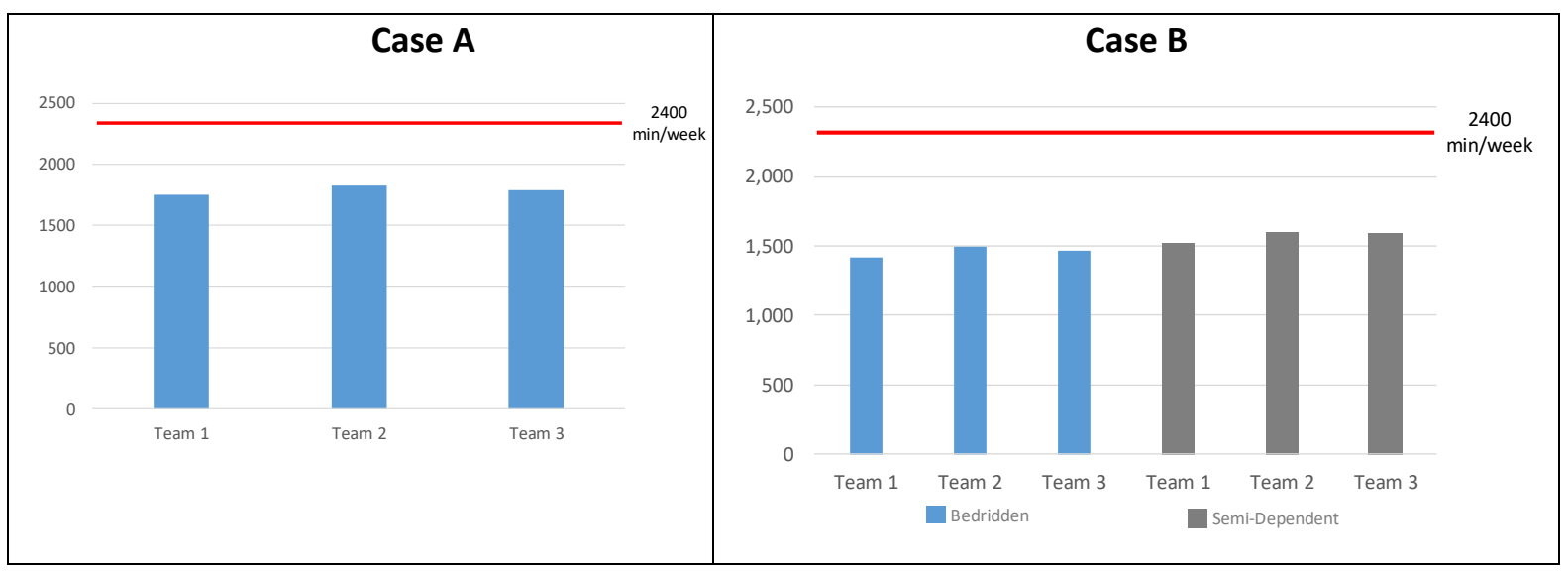

Figure 10: Optimal workload solution (with the lexicographic approach)

Just to illustrate the differences in terms of service areas when both objective functions are applied, Figure 11 shows the areas for each team and for each objective function for case $A$. One can see that patients assigned to each team are quite different between the solutions.

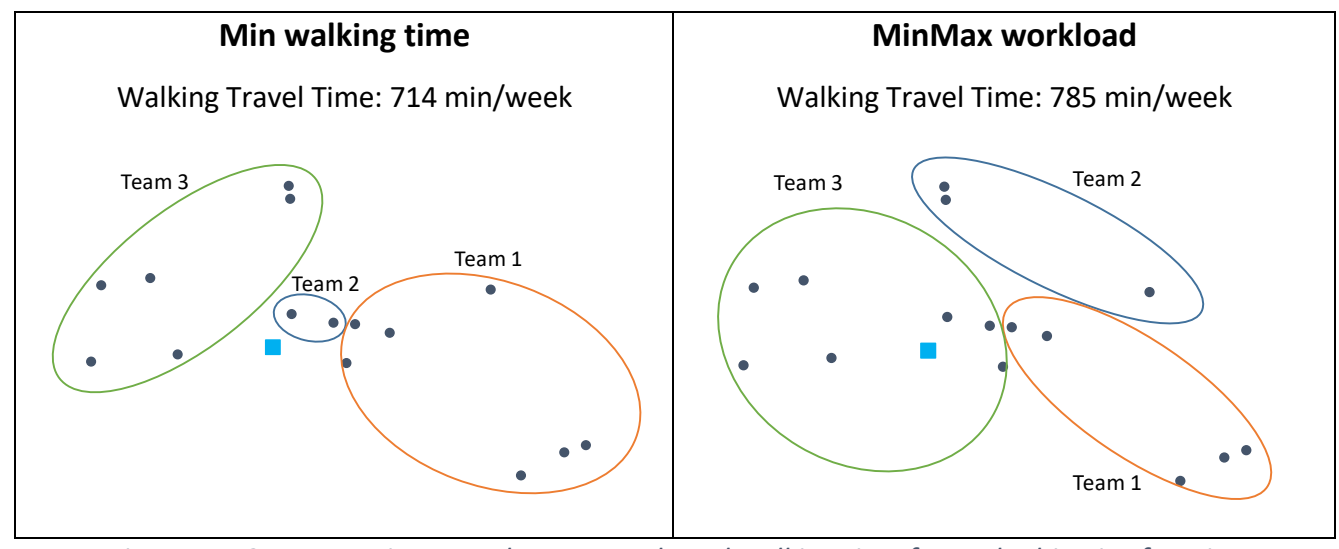

Figure 11: Case A service areas by team and total walking time for each objective function

\section{- Loyalty vs. Non-Loyalty}

Until this point, every plan complies with 1) daily loyalty, i.e., if a patient needs to be visited more than one time per day, those visits are made by the same team; and 2) weekly loyalty, i.e., within a week a patient is always visited by the same team. If daily and weekly loyalties were not imposed by the social 
workers, what would be the impact in terms of travel time? How many new patients could be added? What is the "cost" of the loyalty constraints?

Two scenarios are studied regarding the type of loyalty: one where weekly loyalty is removed, and another where both daily and weekly loyalties are removed. Those results will be compared with the baseline scenario (Scenario 1). Table 7 shows the results for both cases. On one hand, removing the weekly loyalty has little impact in the travel time: for case A it has no impact; for case B semidependent the travel time decreases by $-1.7 \%$, and for the bedridden patients, the travel time increases by $3.4 \%$, but the number of penalties decreases from 10 to 8 . This decrease means that without weekly loyalty is possible to comply with daily loyalty for more bedridden patients. On the other hand, by removing daily loyalty, a more significant impact is observed, especially for case B: for the bedridden patients, the travel time decreases by $26 \%$, and for the semi-dependent it decreases by 9.5\%. Despite this significant impact (especially for the bedridden patients), one needs to be aware that the quality of the service provided can be jeopardized in this scenario. Removing daily loyalty can only be implemented if a good information system supports the homecare operation, since it is essential to the care provided to a patient that the information be well transmitted between caregivers along the day. Moreover, this decrease of $26 \%$ allows the full capacity plan to include three more bedridden patients, instead of two (as in Scenario 2). Likewise, for the semi-dependent patients, the full capacity plan can now include four new patients, instead of three (as in Scenario 2).

Table 7: Impact of relaxing daily and weekly loyalties

\begin{tabular}{l|c|c|c|c|} 
& $\begin{array}{c}\text { Case A } \\
\text { Walking Time }\end{array}$ & $\begin{array}{c}\text { Case B: Bedridden } \\
\text { Travel Time }\end{array}$ & $\begin{array}{c}\text { Case B: Semalties } \\
\text { Travel Time }\end{array}$ \\
Scenario 1 (With Daily and Weekly Loyalty) & 714.2 & 790.2 & 10 & 590.0 \\
Scenario 1.A (Without Weekly Loyalty) & 714.2 & 816.8 & 8 & 580.0 \\
Scenario 1.B (Without Daily and Weekly Loyalty) & 693.9 & 602.4 & - & 525.2
\end{tabular}

To provide further insights on this matter, some benchmark instances were used to test the weekly loyalty regarding the solution quality and the computational time. We use the benchmark instances from the work of Trautsamwieser and Hirsch $(2014)^{2}$, where the planning horizon is of 1 week, as in our work. However, since both problems are different, some simplifications were made to the benchmark instances. For each nurse (in our case, team), we considered the shift length provided for each benchmarking instances, but ignored the qualification level, the speaking language, the breaks, and the fact that some nurses could start their journey from their own houses since those issues are

\footnotetext{
${ }^{2}$ Available at: http://www.wiso.boku.ac.at/en/pwl/forschung/instances/
} 
outside of the scope of our work. For each client, we considered the service duration, the timewindows and the frequency required.

Table 8 shows the results for 19 instances, where the number of teams $(/ A /)$ varies from 2 to 12 , the number of patients $(/ P /)$ varies from 10 to 60 and the planning horizon $(/ T /)$ is always 7 days. Those instances were solved by our full model with weekly loyalty between patient-team (constraint [14]) and without weekly loyalty. A comparison is made regarding the objective function value (OFV) and the computational time (CPU), being the latter limited to 9000 seconds.

It can be observed that the model with weekly loyalty has a worst performance when compared with the results from the model without weekly loyalty. Only four instances were solved to optimality with weekly loyalty, while 17 instances were solved to optimality without weekly loyalty in a short time (a maximum of 1024 seconds is observed at instance 6). Both models failed to provide an integer solution for the largest instances (instances 8 and 9) within the 9000-second limit.

Regarding the solution quality, relaxing the weekly loyalty provides an average improvement of $7.7 \%$, with a minimum of $0.2 \%$ for instance $4 \mathrm{f}$ and a maximum of $21.6 \%$ for instance $4 \mathrm{~d}$. It must be recalled that a fair comparison can only be done when both models provide the optimal solutions, i.e., for instances $2,3,4 \mathrm{~b}$ and $4 \mathrm{j}$, where an average improvement of $7.4 \%$ is observed.

Table 8: Results for benchmarking instances with and without weekly loyalty

\begin{tabular}{|c|c|c|c|c|c|c|c|c|c|c|c|}
\hline \multirow[b]{2}{*}{ Instances } & \multirow[b]{2}{*}{$|A|$} & \multirow[b]{2}{*}{$|P|$} & \multirow[b]{2}{*}{$|T|$} & \multicolumn{3}{|c|}{ With Weekly Loyalty } & \multicolumn{3}{|c|}{ Without Weekly Loyalty } & \multicolumn{2}{|c|}{ Comparation } \\
\hline & & & & OFV1 & GAP (\%) & CPU1 & OFV2 & GAP (\%) & CPU2 & $\frac{(O F V 2-O F V 1)}{O F V 1}$ & $\frac{(C P U 2-C P U 1)}{C P U 1}$ \\
\hline 1 & 2 & 10 & 7 & & Infeasible & & 1091 & 0.0 & 0.3 & - & - \\
\hline 2 & 3 & 15 & 7 & 1795 & 0.0 & 11 & 1646 & 0.0 & 0.7 & $-8.3 \%$ & $-93.6 \%$ \\
\hline 3 & 4 & 20 & 7 & 1964 & 0.0 & 297 & 1925 & 0.0 & 30 & $-2.0 \%$ & $-89.9 \%$ \\
\hline 4 & 6 & 30 & 7 & 3459 & 11.1 & 9000 & 3266 & 0.0 & 28 & $-5.6 \%$ & $-99.7 \%$ \\
\hline $4 a$ & 6 & 30 & 7 & 2172 & 13.1 & 9000 & 2004 & 0.0 & 41 & $-7.7 \%$ & $-99.5 \%$ \\
\hline $4 \mathrm{~b}$ & 6 & 30 & 7 & 2311 & 0.0 & 6444 & 2190 & 0.0 & 29 & $-5.2 \%$ & $-99.5 \%$ \\
\hline $4 c$ & 6 & 30 & 7 & 2407 & 10.3 & 9000 & 2224 & 0.0 & 67 & $-7.6 \%$ & $-99.3 \%$ \\
\hline $4 d$ & 6 & 30 & 7 & 2391 & 18.6 & 9000 & 1874 & 0.0 & 184 & $-21.6 \%$ & $-98.0 \%$ \\
\hline $4 e$ & 6 & 30 & 7 & 2500 & 14.2 & 9000 & 2338 & 0.0 & 27 & $-6.5 \%$ & $-99.7 \%$ \\
\hline $4 f$ & 6 & 30 & 7 & 3450 & 15.0 & 9000 & 3444 & 0.0 & 748 & $-0.2 \%$ & $-91.7 \%$ \\
\hline $4 \mathrm{~g}$ & 6 & 30 & 7 & 2833 & 17.5 & 9000 & 2599 & 0.0 & 80 & $-8.3 \%$ & $-99.1 \%$ \\
\hline $4 \mathrm{~h}$ & 6 & 30 & 7 & 2861 & 16.1 & 9000 & 2757 & 0.0 & 46 & $-3.6 \%$ & $-99.5 \%$ \\
\hline $4 i$ & 6 & 30 & 7 & 2676 & 13.5 & 9000 & 2529 & 0.0 & 112 & $-5.5 \%$ & $-98.8 \%$ \\
\hline $4 \mathrm{j}$ & 6 & 30 & 7 & 2375 & 0.0 & 6295 & 2039 & 0.0 & 15 & $-14.1 \%$ & $-99.8 \%$ \\
\hline 5 & 7 & 35 & 7 & 3177 & 14.6 & 9000 & 2977 & 0.0 & 36 & $-6.3 \%$ & $-99.6 \%$ \\
\hline 6 & 8 & 40 & 7 & & & & 3047 & 0.0 & 1024 & - & - \\
\hline 7 & 9 & 45 & 7 & 4006 & 19.6 & 9000 & 3466 & 0.0 & 152 & $-13.5 \%$ & $-98.3 \%$ \\
\hline 8 & 10 & 50 & 7 & & & & & & & - & - \\
\hline 9 & 12 & 60 & 7 & & & & & & & - & - \\
\hline
\end{tabular}

OFV: Objective Function Value

GAP (\%): Percentage deviation between the upper bound and the lower bound in the branch-and-cut algorithm of the CPLEX after the computational time limit has been reached CPU: Computational Time (in seconds)

\subsection{The caregiver assignment model}


At the previous section, we obtained a scheduling and a routing plan for each team, for each day of the week, for both case studies. In this section, we must define the weekly allocation of each caregiver to a team. The time horizon is one of the caregiver assignment model sets (set $T$ ) and its cardinality is not known a priori. Therefore, we use an iterative process through which the number of weeks is increased until a feasible solution is found. Notice that we aim at finding the smallest time horizon allowing for a rolling horizon scheme for the allocation of caregivers to teams.

Case A has six caregivers to be assigned to three teams of two people. In case B, nine caregivers are to be assigned to six teams, where three teams are composed by two people and three are teams of just one person. For the latter, we assume three fictitious caregivers (see section 4.2.2).

A feasible solution is reached with a time horizon of 8 weeks for case $A$ and 12 weeks for case $B$. This means the solutions found can be repeated after 8 weeks (for case A) and 12 weeks (for case B), satisfying all the constraints. Figure 12 depicts case A solution, wherein each caregiver is illustrated by a different colour. All requests made by the social workers are met: one caregiver is on the same team only for two or three weeks in a row, all 15 different pairs of caregivers are present in the solution and all caregivers (all colours) are also present in all teams.

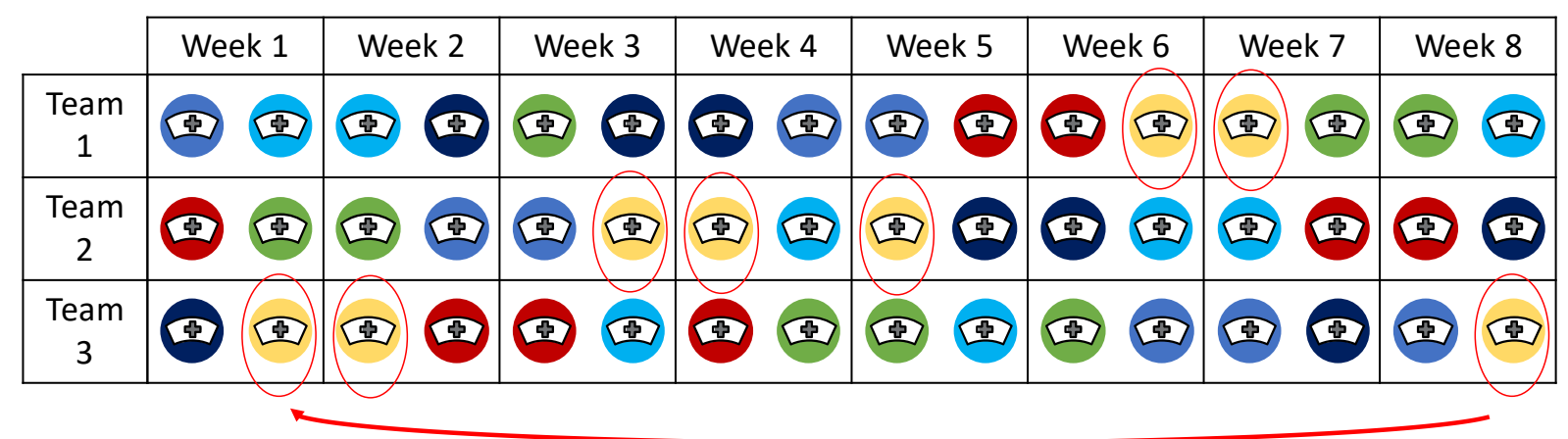

Figure 12: Allocation of caregivers to teams over a time horizon of 8 weeks for case A (each caregiver is represented by a different colour)

The solution for case $B$ is shown in Figure 13. Each of one of the nine real caregivers is assigned to one of the six teams. For example, caregiver \#1 is assigned to team 1 during the two first weeks and then is assigned to team 2, where he/she stays for two more weeks, and so on. The caregivers assigned to the semi-dependent teams (grey rows) do not stay at the same team for more than two consecutive weeks (see for example caregiver \#4 is assigned to team 5 at week 4 and 5 , but at week 6 is assigned to team 4). All caregivers rotate among every team. Each caregiver stays 8 weeks with the bedridden teams and 4 weeks with the semi-dependent teams during a planning horizon of 12 weeks, satisfying constraint [ 40$]$. 


\begin{tabular}{|c|c|c|c|c|c|c|c|c|c|c|c|c|}
\hline & $\begin{array}{c}\text { Week } \\
1\end{array}$ & $\begin{array}{c}\text { Week } \\
2\end{array}$ & $\begin{array}{c}\text { Week } \\
3\end{array}$ & $\begin{array}{c}\text { Week } \\
4\end{array}$ & $\begin{array}{c}\text { Week } \\
5\end{array}$ & $\begin{array}{c}\text { Week } \\
6\end{array}$ & $\begin{array}{c}\text { Week } \\
7\end{array}$ & $\begin{array}{c}\text { Week } \\
8\end{array}$ & $\begin{array}{c}\text { Week } \\
9\end{array}$ & $\begin{array}{c}\text { Week } \\
10\end{array}$ & $\begin{array}{c}\text { Week } \\
11\end{array}$ & $\begin{array}{c}\text { Week } \\
12\end{array}$ \\
\hline Team 1 & $\begin{array}{ll}\# 2 & \# 1 \\
\text { (क) (5) }\end{array}$ & $\begin{array}{ll}\# 7 & \# 1 \\
\text { (क्) (क) }\end{array}$ & $\begin{array}{ll}\# 8 & \# 7 \\
\text { (क) }\end{array}$ & $\begin{array}{l}\# 8 \text { \#3 } \\
\text { (5) (5) }\end{array}$ & $\begin{array}{l}\# 6 \text { \#3 } \\
\text { का का क }\end{array}$ & $\begin{array}{ll}\# 9 & \# 6 \\
\text { (क) क }\end{array}$ & $\begin{array}{ll}\# 7 & \# 6 \\
\text { का } & \text { क }\end{array}$ & $\# 7$ \# & $\begin{array}{ll}\# 7 & \# 4 \\
\text { (4) }\end{array}$ & $\begin{array}{ll}\# 5 & \# 4 \\
\Leftrightarrow & 0\end{array}$ & 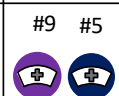 & \#9 \\
\hline & 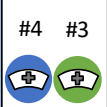 & $\begin{array}{l}\# 9{ }^{\# 4} \\
\text { (\$) }(5)\end{array}$ & $\begin{array}{ll}\# 4 & \# 1 \\
\text { (5) } 5 \text { (5) }\end{array}$ & $\begin{array}{ll}\# 5 & \# 1 \\
\text { (5) } & \text { (5) }\end{array}$ & $\begin{array}{l}\# 5 \# 2 \\
\text { (5) 딴 }\end{array}$ & \#8 & $\begin{array}{l}\# 9 \text { \#8 } \\
\text { क क }\end{array}$ & 한 & 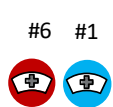 & $\begin{array}{ll}\# 6 & \# 2 \\
\text { () }\end{array}$ & (4) \#2 & (क) $\# 3$ \\
\hline Tea & $\begin{array}{l}\# 6 \text { क } \# 5 \\
\text { का का }\end{array}$ & \#3 & $\begin{array}{l}\# 9 \text { \#3 } \\
\text { (क) का }\end{array}$ & $\begin{array}{ll}\# 9 & \# 7 \\
\text { क }\end{array}$ & $\begin{array}{ll}\# 9 & \# 1 \\
\text { (क) (क) }\end{array}$ & \#3 \#1 & $\begin{array}{l}\# 3 \quad \# 2 \\
\text { (5) 화 }\end{array}$ & $\begin{array}{ll}\# 4 & \# 2 \\
\text { (5) क्ञ }\end{array}$ & 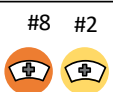 & (5) $\# 8$ & 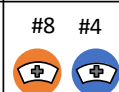 & (क) $\# 4$ \\
\hline Tean & $\begin{array}{l}\# 10 \text { \#8 } \\
\text { (45) (5) }\end{array}$ & 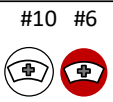 & $\begin{array}{l}\# 10 \# 2 \\
\$ \text { क }\end{array}$ & $\begin{array}{l}\# 10 \# 6 \\
\text { (4) (क) }\end{array}$ & $\begin{array}{l}\# 10 \# 7 \\
\text { (4) (क) }\end{array}$ & $\begin{array}{l}\# 10 \# 4 \\
\text { (5) (5) }\end{array}$ & $\begin{array}{l}\# 10 \# 5 \\
\text { (5) }\end{array}$ & $\begin{array}{l}\# 10 \# 1 \\
\text { (5) }\end{array}$ & $\begin{array}{l}\# 10 \# 3 \\
\text { (화 (5) }\end{array}$ & $\begin{array}{l}\# 10 \# 9 \\
\text { (क्) क्ञ }\end{array}$ & $\begin{array}{l}\# 10 \# 1 \\
\text { (4) (5) }\end{array}$ & $\begin{array}{l}\# 10 \# 5 \\
\text { (क क क }\end{array}$ \\
\hline Tea & $\begin{array}{l}\# 11 \text { \#7 } \\
\text { (5) (5) }\end{array}$ & (4) \#8 & \#11 \#6 & 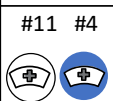 & $\begin{array}{l}\# 11 \quad \# 4 \\
\text { (5) (5) }\end{array}$ & $\begin{array}{l}\# 11 \text { \#2 } \\
\text { (5) क्ष }\end{array}$ & 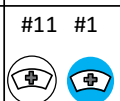 & \#11 \#9 & 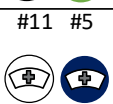 & $\begin{array}{l}\# 11 \# 7 \\
\text { (4) का }\end{array}$ & 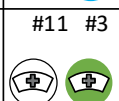 & $\begin{array}{l}\# 11 \text { \#8 } \\
\text { (45) क6 }\end{array}$ \\
\hline & $\# 12$ \#9 & $\begin{array}{l}\# 12 \# 2 \\
\text { (4) क्ष }\end{array}$ & $\begin{array}{l}\# 12 \text { \#5 } \\
\text { (क) का }\end{array}$ & $\begin{array}{l}\# 12 \# 2 \\
\text { (45) क्ष }\end{array}$ & $\begin{array}{l}\# 12 \# 8 \\
\text { (5) (5) }\end{array}$ & $\begin{array}{l}\# 12 \# 7 \\
\text { (5) (5) }\end{array}$ & 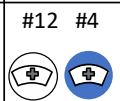 & $\begin{array}{l}\# 12 \# 3 \\
\text { (5) }\end{array}$ & $\# 12$ \#9 & $\begin{array}{l}\# 12 \# 3 \\
\text { (\$) } \$\end{array}$ & (4) $\# 12$ (5) & $\# 12 \quad \# 1$ \\
\hline
\end{tabular}

Bedridden

Wemi-Dependent (5)

Figure 13: Allocation of caregivers to teams over a time horizon of 12 weeks for case $B$

\section{Conclusions}

Two Portuguese home social care service providers motivated this paper, where the social workers in charge pursue a scheduling plan for their caregivers that should impose loyalty within a day and within a week between patient and caregiver, but non-loyalty between weeks and between team members. The loyalty feature is very common in home care context. However, the non-loyalty feature has never been addressed by the academia, to our best knowledge, but is present in practice. The reasons behind the non-loyalty are related to social relationship issues between patients and caregivers, between caregivers when forming teams, and to prevent job-related injuries and illnesses. Moreover, both services face frequent changes in the set of patients to visit and a long waiting list. When a patient leaves the system, the "freed space" should be assigned to a new patient without disturbing the already visited patients' schedules. Again, the planning of a home social care problem assuming a previous plan has not been addressed in the literature.

In this work, we address both the caregiver assignment problem and the scheduling and routing definition having, for the former, a specific set of rules to comply with and, for the latter, a previous plan that should be taken into account. With the introduction of the concept of "teams", we are able to break down our problem into two independent subproblems without losing solution optimality. Two new MILP models are proposed: the multi-objective team scheduling and routing model and the caregiver assignment model with a rolling horizon. 
The caregiver assignment model assures the non-loyalty between weeks by assigning caregivers to teams. Eight and twelve weeks are the smallest number of weeks that allows a feasible solution of a rolling horizon scheme with the requested rules, for case $A$ and $B$, respectively.

In the scheduling and routing model, daily and weekly loyalty are assured, and the solution obtained is to be repeated every week until some patient leaves the system. When it happens, a new patient from the waiting list is admitted that (optimally) fits the previous visiting plan with controlled changes in the previous starting times of visits and with a minimum increase in the caregivers total travelling times. By removing some constraints, the model can optimize the patients' visiting schedule and routing, assuming that no previous plan exists. As main results, we are able to reduce $23 \%$ in walking time for case $A$ and $6 \%$ in car travelling time for case $B$ when comparing with the current solution (Scenario 1). This reduction allows an increase in the number of patients that can be served by both organizations without increasing the number of caregivers (Scenario 2). The simulations pertaining to patients that leave the system showed that the model is an adequate tool to support the social workers' decision making in regards to the selection of new patients and the scheduling of visits. . Moreover, the current patients will not have their schedules changed more than desired (Scenario 3). When balancing workload, more equitable schedules for each team are achieved with small increases in travel time. Lastly, it was also assessed the impact of imposing daily and weekly loyalty. The main conclusions are: relaxing only weekly loyalty does not impact much the solution quality for the real case-studies, while for the benchmarking instances the impact in on average of 7\%; relaxing both daily and weekly loyalty at the same time led to a considerable reduction on the travel time and, consequently, increased the ability to assist more patients in the real case-studies. Regarding the computational performance over the benchmarking instances, the model with non-loyalty constraints performs better as expected. Nonetheless, one should not forget that managing a non-loyalty service will demand much more coordination between the social workers and the caregivers so that the needs of the patients are adequately fulfilled.

As for future work, two main ideas will be explored. Firstly, the decomposition approach could be improved to account for both patients' typologies simultaneously. We aim at investigating the benefits in term of solution quality if the same caregiver is allowed to visit both semi-dependent patients and bedridden patients on the same day. Second, the model can also be extended to deal with uncertainty. Several uncertainty sources exist in home care problems, such as the duration of the service as we are dealing with a high-level customer contact service which has a major impact on the duration of the service; the absent of caregivers on a given day (e.g., one may get sick); patients may be absent from their homes (to go to doctor's appointments or may go to relatives' home for some days). These are 
just few examples of sources of uncertainty that motivate a stochastic approach, which despite being a common practice in other VRP applications, has not yet caught the attention in HC problems (Fikar and Hirsch, 2017).

\section{Acknowledgment}

This work was partially supported by the Fundação para a Ciência e a Tecnologia (Portuguese Foundation for Science and Technology) through the project UID/MAT/00297/2019 (Centro de Matemática e Aplicações)

\section{References}

Akjiratikarl, C., Yenradee, P., \& Drake, P. R. (2007). PSO-based algorithm for home care worker scheduling in the UK. Computers \& Industrial Engineering, 53(4), 559-583.

Begur, S. V., Miller, D. M., \& Weaver, J. R. (1997). An integrated spatial DSS for scheduling and routing home-health-care nurses. Interfaces, 27(4), 35-48.

Bertels, S., \& Fahle, T. (2006). A hybrid setup for a hybrid scenario: combining heuristics for the home health care problem. Computers \& Operations Research, 33(10), 2866-2890.

Braekers, K., Hartl, R. F., Parragh, S. N., \& Tricoire, F. (2016). A bi-objective home care scheduling problem: Analyzing the trade-off between costs and client inconvenience. European Journal of Operational Research, 248(2), 428-443.

Carello, G., \& Lanzarone, E. (2014). A cardinality-constrained robust model for the assignment problem in home care services. European Journal of Operational Research, 236(2), 748-762.

Cattafi, M., Herrero, R., Gavanelli, M., Nonato, M., \& Malucelli, F. (2012). Improving quality and efficiency in home health care: An application of constraint logic programming for the Ferrara NHS unit. In LIPIcs-Leibniz International Proceedings in Informatics (Vol. 17). Schloss Dagstuhl-LeibnizZentrum fuer Informatik.

Cheng, E., \& Rich, J. L. (1998). A home health care routing and scheduling problem. Houston, Texas.

Cordeau, J., Laporte, G., Savelsbergh, M.W.P., Vigo, D., (2007). Vehicle Routing, in: Barhart, C., Laporte, G. (Eds.), Handbook in OR\&MS. Elsevier B.V., 367-428.

European Commission (2014). The 2015 Ageing Report: Underlying Assumptions and Projection 
Methodologies. Retrieved from:

http://ec.europa.eu/economy_finance/publications/european_economy/2014/pdf/ee 8_en.pdf.

Eveborn, P., Flisberg, P., \& Rönnqvist, M. (2006). Laps Care-an operational system for staff planning of home care. European Journal of Operational Research, 171(3), 962-976.

Eveborn, P., Rönnqvist, M., Einarsdóttir, H., Eklund, M., Lidén, K., \& Almroth, M. (2009). Operations research improves quality and efficiency in home care. Interfaces, 39(1), 18-34.

Fikar, C., \& Hirsch, P. (2017). Home health care routing and scheduling: A review. Computers \& Operations Research 77, 86-95.

Gamst, M., \& Jensen, T. S. (2012). A branch-and-price algorithm for the long-term home care scheduling problem. In Operations research proceedings 2011(pp. 483-488). Springer, Berlin, Heidelberg.

Gershon, R.R.M., Pogorzelska, M., Qureshi, K.A., et al. (2008). Home Health Care Patients and Safety Hazards in the Home: Preliminary Findings. In Henriksen, K., Battles, J.B., et al. (eds.) Advances in Patient Safety: New Directions and Alternative Approaches (Vol. 1: Assessment). Agency for Healthcare Research and Quality. Rockville (MD).

INE (2015). Dia Mundial da população. Retrieved from: https://www.ine.pt/ngt_server/attachfileu.jsp?look_parentBoui=232024193\&att_displ ay=n\&att_download=y.

Koeleman, P. M., Bhulai, S. and van Meersbergen, M. (2012). Optimal patient and personnel scheduling policies for care-at-home service facilities. European Journal of Operational Research, 219 (3), 557-563.

Lanzarone, E., \& Matta, A. (2014). Robust nurse-to-patient assignment in home care services to minimize overtimes under continuity of care. Operations Research for Health Care, 3(2), 48-58.

Lin, M., Chin, K.S., Wang X. and Tsui, K.L. (2016). The therapist assignment problem in home healthcare structures. Expert Systems with Applications, 62, 44-62.

Liu, R., Xie, X. and Garaix, T. (2014). Hybridization of tabu search with feasible and infeasible local searches for periodic home health care logistics. Omega, 47, 17-32.

Mankowska, D. S., Meisel, F. \& Bierwirth, C. (2014). The home health care routing and scheduling problem with interdependent services. Health Care Management Science, 17, 15-30.

Nickel, S., Schröder, M., \& Steeg, J. (2012). Mid-term and short-term planning support for home health care services. European Journal of Operational Research, 219(3), 574-587. 
Olinski, C. \& Norton, C.E., (2017). Implementation of a Safe Patient Handling Program in a Multihospital Health System from Inception to Sustainability: Successes over 8 Years and Ongoing Challenges. Workplace Health \& Safety (published online)

Rasmussen, M. V., Justesen, T., Dohn, A. \& Larsen, J. (2012). The Home Care Crew Scheduling Problem: Preference-based visit clustering and temporal dependencies. European Journal of Operational Research, 219, 598-610.

Trautsamwieser, A., \& Hirsch, P. (2011). Optimization of daily scheduling for home health care services. Journal of Applied Operational Research, 3(3), 124-136.

Trautsamwieser, A., \& Hirsch, P. (2014). A Branch-Price-and-Cut approach for solving the mediumterm home health care planning problem. Networks, 64(3), 143-159. 